\title{
Aleaciones de Al-Cr-Zr para aplicaciones a altas temperaturas ${ }^{(*)}$
}

\author{
T. Gerique ${ }^{(*)}$, A. Brahmi ${ }^{(*)}$, M. Lieblich ${ }^{(*)}$ y M. Torralba ${ }^{(*)}$
}

\begin{abstract}
Resumen Se ha revisado la bibliografía existente sobre las aleaciones Al-Cr-Zr para alta temperatura. Además de una breve reseña del desarrollo histórico y una descripción de los fundamentos de estas aleaciones de aluminio-metal de transición, se comentan los métodos de obtención y las bases de la solidificación rápida, así como diversos métodos de consolidación. También se indican los mecanismos de refuerzo de estas aleaciones mediante intermetálicos incoherentes y se revisa en detalle la estabilidad térmica y las propiedades mecánicas de las mismas, incluido su comportamiento en fluencia.
\end{abstract}

Palabras clave: Al-Cr-Zr. Alta temperatura. Solidificación rápida. Consolidación. Propiedades mecánicas.

\section{Al-Cr-Zr alloys for high temperature applications}

\begin{abstract}
The literature on Al-Cr-Zr alloys for high temperature applications is revised. In addition to a short historical review and a description of the fundamental aspects of these aluminium-transition metal alloys, comments on processing methods, including rapid solidification and various consolidation techniques are considered. The strengthening mechanisms, mainly due to incoherent intermetallic particles, the thermal stability and the mechanical properties of the $\mathrm{Al}-\mathrm{Cr}-\mathrm{Zr}$ alloys, including creep behaviour, are thoroughly examined.
\end{abstract}

Keywords: Al-Cr-Zr. High temperature. Rapid solidification. Consolidation. Mechanical properties.

\section{ANTECEDENTES}

La industria aeronáutica es muy dinámica en investigación, y el desarrollo de nuevos materiales metálicos para este sector no constituye una excepción, especialmente en lo que se refiere al aluminio (1). En un plazo no superior a ocho años es preciso desarrollar un avión civil supersónico completamente nuevo que pueda reemplazar al ya muy veterano Concorde. Para ello, entre otras muchas cosas, es preciso desarrollar materiales metálicos ligeros y resistentes a temperaturas relativamente altas. Para aplicaciones a temperatura ambiente, $T_{\mathrm{A}}$, y hasta $100{ }^{\circ} \mathrm{C}$, se dispone de excelentes aleaciones de aluminio (densidad $2.700-2.900 \mathrm{~kg} \mathrm{~m}^{-3}$ ) preparadas por metalurgia clásica. A partir de $100-150{ }^{\circ} \mathrm{C}$, estas aleaciones se degradan rápidamente. Las pro-

$(\bullet \quad$ Trabajo recibido el día 18 de diciembre de 1997 y aceptado en su forma final el 5 de marzo de 1998.

(*) Dpto. de Metalurgia Física. Centro Nacional de Investigaciones Metalúrgicas, CENIM (CSIC). Avda. Gregorio del Amo, 8. 28040-Madrid (España). piedades mecánicas, y entre ellas el límite elástico $(Y S)$ de las principales aleaciones de aluminio para aeronáutica disminuyen muy rápidamente a partir de $100-150{ }^{\circ} \mathrm{C}$, incluida la aleación 7075 (Al-Zn$\mathrm{Mg}-\mathrm{Cu}$ ) en estado $\mathrm{T} 6$, que es una de las de mayor resistencia a $T_{\mathrm{A}}$ y la más utilizada en construcción aeronáutica. Por encima de estas temperaturas y hasta $450{ }^{\circ} \mathrm{C}$ hay que recurrir a las aleaciones de titanio (densidad $4.400-4.600 \mathrm{~kg} \mathrm{~m}^{-3}$ ) y a temperaturas aún más altas a los aceros, a las superaleaciones y a los compuestos intermetálicos, de densidad muy superior. Dado el alto precio y la escasez del titanio es muy importante el desarrollo de aleaciones de aluminio que puedan utilizarse con garantías por encima de $100^{\circ} \mathrm{C}$.

La puesta a punto de aleaciones de aluminio que puedan utilizarse en el intervalo de temperaturas de 100 a $350{ }^{\circ} \mathrm{C}\left(0,4-0,67 T_{\mathrm{f}}\right.$, donde $T_{\mathrm{f}}=933 \mathrm{~K}$ es la temperatura de fusión del aluminio puro) presenta varias ventajas respecto a las de titanio: a) reducción del precio del material y del coste de fabricación; b) reducción de la masa de las piezas y, en consecuencia, cuando son piezas giratorias, reducción del 
momento de inercia, y c) reducción del consumo de titanio considerado material estratégico.

Un objetivo para su posible utilización sería conseguir aleaciones de aluminio que, después de tratadas $1.000 \mathrm{~h}$ a la temperatura de servicio, entre 100 y $350{ }^{\circ} \mathrm{C}$, tuvieran igual límite elástico específico que la aleación Ti-4Al-6V con el mismo tratamiento (Fig. 1) (todas las composiciones de las aleaciones están dadas en tanto por ciento en masa), haciendo posible la sustitución de éstas por las de aluminio en ese intervalo de temperaturas.

Para la producción de estos materiales es preciso recurrir a técnicas de solidificación rápida (SR) y de pulvimetalurgia. Esto, sin embargo, no constituye un obstáculo económico para su desarrollo ya que el precio del producto final es, aún así, inferior al del mismo producto realizado con aleaciones de titanio.

Las aplicaciones industriales para altas temperaturas de los materiales de base aluminio se encuentran principalmente en la industria aeronáutica: rotores de compresión de turbinas de gas (2-4), válvulas, cárteres, alerones, piezas de misiles o fuselajes de aviones que han de volar a altas velocidades (Mach 2 ó 3) (2, 5 y 6), así como tubos de conducción de aire caliente en sistemas para eliminar el hielo de las alas y de las barquillas de aviones subsónicos, etc. (7). En la industria del automóvil su aplicación permitiría aumentar la vida en servicio de pistones y, tal vez, de bielas, así como su utilización a temperatura más elevada (5 y 8). También servirían para la fabricación de rotores de compresión de motores auxiliares (2).

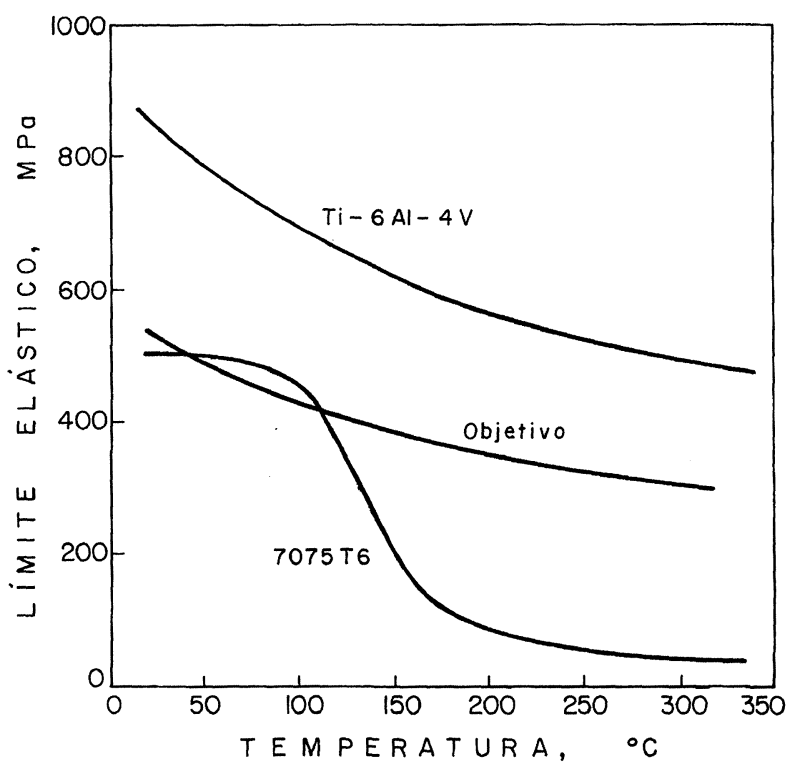

FIG. 1.- Objetivo del límite elástico específico para aleaciones de aluminio.

FIG. 1.- Target of the yield stress in aluminium alloys.

\section{MECANISMOS DE REFORZAMIENTO}

Las aleaciones de aluminio de alta resistencia utilizadas a $T_{\mathrm{A}}$ son, por lo general, del tipo denominado aleaciones tratables (9). En estas aleaciones, los elementos aleantes se disuelven mediante un tratamiento térmico adecuado y luego se templan hasta $T_{\mathrm{A}}$ para conseguir una solución sólida sobresaturada. A continuación, se da un tratamiento térmico que produce la precipitación. Las propiedades mecánicas así obtenidas se deben principalmente a la formación de precipitados coherentes o semicoherentes que, por lo general, son metaestables. Los precipitados se forman a partir de los elementos: cobre, silicio, manganeso, magnesio y zinc, y de otros que aparecen en menor proporción tales como hierro y cromo. Sin embargo, dado que los cinco elementos mencionados en primer lugar son muy solubles en aluminio y su difusibidad es grande, al someter las aleaciones a altas temperaturas, los precipitados o bien se disuelven, o bien crecen por maduración de Ostwald perdiendo su coherencia, con la 'consiguiente pérdida de propiedades mecánicas de la aleación. Por tanto, es necesario buscar otros métodos de reforzamiento para las aleaciones de alta temperatura.

Los mecanismos de reforzamiento más frecuentes, además del endurecimiento por trabajado en frío, son los relacionados con la solución sólida, precipitados y dispersoides, así como el efecto de las fronteras de grano y de subgrano. El endurecimiento por efecto de la solución sólida requiere una gran solubilidad del aleante y que los átomos de éste tengan una diferencia de tamaño considerable con los de aluminio para que introduzcan grandes distorsiones en la red. La efectividad de los dispersoides depende de que estén finamente distribuidos. Dependiendo de su tamaño, espaciado y grado de coherencia, las partículas son cizalladas o rodeadas por las dislocaciones móviles durante la deformación plástica.

Si las partículas son cizallables, en general precipitados coherentes o semicoherentes, el reforzamiento producido depende de las propiedades intrínsecas de las partículas, que producen tensiones elásticas a su alrededor dificultando así el movimiento de las dislocaciones. La tensión de fluencia $\left(\sigma_{\mathrm{y}}\right)$ se puede representar por una ecuación del tipo:

$$
\sigma_{\mathrm{y}}=c f^{\mathrm{m}} r^{\mathrm{p}}
$$

donde $f$ es la fracción de volumen de partículas y $r$ su radio. Los exponentes $m$ y $p$ son positivos y el endurecimiento aumenta con la fracción de volumen y el tamaño de las partículas. El parámetro $c$ es una constante de la aleación que depende del grado de coherencia entre las partículas y la matriz, de la relación entre sus módulos de elasticidad, del grado de orden de las partículas y de la energía de la intercara. 
Cuando las partículas son rodeadas por las dislocaciones, el reforzamiento producido es independiente de las propiedades de las partículas y la tensión de fluencia de la aleación viene dada por la relación de Orowan modificada:

$$
\sigma_{\mathrm{y}}=\sigma_{0}+0,8 \mathrm{G} b / \mathrm{L}
$$

donde $\sigma_{0}$ es la tensión de fluencia de la matriz, $G$ el módulo de cizalla de la misma, $b$ el vector Burgers y $L$ el espaciado entre partículas. Para una fracción de volumen de partículas constante, el reforzamiento disminuye al aumentar el tamaño de las partículas o, lo que es lo mismo, al aumentar su espaciado. Conseguir este reforzamiento por partículas no cizallables tiene serias dificultades utilizando métodos de metalurgia clásica, ya que es muy difícil obtener una dispersión homogénea de partículas pequeñas, duras, incoherentes, próximas entre sí y térmicamente estables.

Las fronteras de grano y subgrano tienden a impedir el movimiento de las dislocaciones y son una importante fuente de reforzamiento. Además, el afinamiento de grano mejora la ductilidad, la tenacidad a la fractura, la resistencia a la corrosión y la resistencia a la iniciación de grietas por fatiga. El efecto del tamaño de grano sobre la tensión de fluencia se describe generalmente por la relación de Hall-Petch:

$$
\sigma_{\mathrm{y}}=\sigma_{0}+k d^{-1 / 2}
$$

donde $\sigma_{0}$ y $k$ son constantes del material, y $d$ el tamaño medio de grano. Una relación similar se ha establecido para el endurecimiento por subgranos o celdas. En este caso, $k$ suele ser menor y el exponente de $d$ suele tomar el valor 1 .

En resumen, dado que las aleaciones clásicas de aluminio no son útiles a temperaturas elevadas a causa de la alta difusividad de la mayoría de los aleantes que entran en su composición, es preciso recurrir a otras familias de aleaciones, donde los principales factores de reforzamiento sean los debidos, bien a la tensión de Orowan, bien a la de HallPetch, o a ambas.

\section{SOLIDIFICACIÓN RÁPIDA}

Como ya se ha mencionado, la utilización de aleaciones de aluminio hasta $350{ }^{\circ} \mathrm{C}$ supone la puesta a punto de nuevas familias de aleaciones con pequeños dispersoides endurecedores, térmicamente estables y homogéneamente repartidos. Para que estos dispersoides sean estables a altas temperaturas, los elementos de adición que entran en su composición deben difundir muy lentamente en la matriz de aluminio, y los precipitados a que dan lugar deben tener baja energía de intercara con la matriz, lo que reduce su tendencia a crecer por maduración. Los metales de transición, así como las tierras raras, satisfacen bien estas condiciones (1012). Sin embargo, las aleaciones de aluminio-metal de transición preparadas por metalurgia clásica contienen grandes precipitados que las hacen extraordinariamente frágiles, debido a que los metales de transición y las tierras raras son casi insolubles en aluminio en estado sólido (13), por lo que al solidificar la aleación se separan del metal base en forma de grandes segregaciones.

Así pues, es necesario recurrir a técnicas de solidificación rápida que amplían el campo de solubilidad sólida y, en consecuencia, a la pulvimetalurgia. Otra opción es el aleado mecánico, que permite producir aleaciones de sistemas inmiscibles en estado sólido lo que, sin embargo, no evita recurrir a la pulvimetalurgia (14 y 15). Los métodos de SR tienen muchas variantes que afectan a la estructura y propiedades del material, así como a las reacciones que tienen lugar durante su tratamiento térmico.'Las principales variaciones que introducen respecto a técnicas como la colada continua, son: a) extensión de la solubilidad en estado sólido; b) afino del tamaño de grano; c) modificación del esquema de segregación y d) formación de fases metaestables.

Para conseguir la SR tiene que actuarse sobre pequeñas masas de material o, al menos, con una de sus dimensiones pequeña, de modo que pueda extraerse el calor con rapidez. Al poner una masa de metal fundido en contacto con un sumidero eficiente de calor, el líquido se enfría, produciendo un fuerte subenfriamiento, hasta que aparecen puntos de nucleación. A partir de ellos crecen los cristales hasta ocupar toda la masa líquida, ya que el calor latente de solidificación es absorbido por el metal líquido, si éste está suficientemente subenfriado. Los factores que intervienen en el proceso tales como la velocidad de enfriamiento, el subenfriamiento y la frecuencia de nucleación a cada temperatura están íntimamente relacionados y dependen a su vez de la composición química de la aleación y de las condiciones del proceso. Los métodos para conseguir la SR son muchos, aunque los más prácticos son la atomización y la colada sobre rueda o melt spinning, así como la colada de flujo laminar (CFL) o planar flow casting. El primero es el más viable económicamente aunque no alcanza las velocidades de solidificación más rápidas. En investigación se han utilizado mucho las técnicas de obtención de copos o splats que, en general, dan velocidades de solidificación muy altas.

Las partículas de polvo obtenidas por atomización muestran microestructuras diversas dependiendo de la velocidad de enfriamiento, que pueden variar desde una solución sólida sin partición hasta 
una microestructura de dendritas gruesas. En el caso de aleaciones de aluminio-metal de transición existen estudios que indican las condiciones necesarias para alcanzar la máxima resistencia mecánica a alta temperatura, tales como una alta fracción en volumen de dispersoides intermetálicos, un tamaño pequeño y una distribución homogénea de los mismos, así como una estabilidad térmica elevada de las fases precipitadas (16-18). Todo esto contribuye a aumentar la tensión de Orowan y a anclar las fronteras de grano, impidiendo así su crecimiento por recristalización.

\section{CONSOLIDACIÓN}

Mediante la SR se puede actuar sobre una gran variedad de líquidos metálicos para obtener materiales con una estructura cristalina adecuada y buenas propiedades, aunque de difícil aplicación práctica, ya que estos se presentan en forma de polvos o de cintas que deben ser posteriormente pulverizadas. Por ello, es necesario consolidar los polvos. Los dos procesos más corrientes son la extrusión y la compactación isostática en caliente. Esta última se aplica poco en el caso del aluminio, puesto que las partículas de polvo siempre están recubiertas de una fina capa de alúmina (19 y 20), dada la gran reactividad del aluminio con el oxígeno, lo que dificulta los procesos de difusión que dan lugar a la consolidación. En general, se recurre a la extrusión que introduce tensiones de cizalla que rompen la capa de alúmina. En la práctica, incluso en el caso de las cintas obtenidas por temple sobre rueda o por colada de flujo laminar, su consolidación resulta más efectiva por extrusión de polvos procedentes de cintas previamente trituradas. También se han empleado métodos que consisten en una precompactación de los polvos y posterior deformación, en general por laminación.

Los polvos no se pueden extruir sin una preparación previa, que puede llevarse a cabo por dos vías: a) en la primera se desgasifican en un recipiente que luego es sometido a extrusión en caliente. Todo ello requiere temperaturas relativamente altas durante tiempos de 1 a $3 \mathrm{~h}$, lo que puede producir transformaciones no deseadas de la microestructura; b) en la segunda se precompactan los polvos sin desgasificar hasta densidades que permitan su manejo sin que el tocho se desmorone. Antes de extruir es preciso un precalentamiento a la temperatura de extrusión.

En el caso de la extrusión de polvo de aluminio, la capa de óxido que rodea a cada partícula se rompe durante la extrusión permitiendo la soldadura de las distintas partículas. Esta rotura se produce por dos caminos: por fricción de unas partículas con otras y por la fractura de la capa de óxido, menos dúctil, al deformarse las partículas. Las partículas de óxido pueden permanecer en la frontera entre las partículas de polvo o introducirse dentro de ellas. Con respecto a las propiedades mecánicas, el primer caso es desfavorable, mientras que el segundo refuerza el material.

Los materiales extruidos pueden presentar características muy distintas dependiendo de: la estabilidad de las microestructuras de SR frente a la temperatura; las condiciones de la consolidación; el espectro de tamaños del polvo y la velocidad de solidificación en función de sus tamaños.

Dado que las estructuras de SR suelen ser poco estables frente a la temperatura, los tratamientos térmicos implicados en la desgasificación y en la extrusión pueden dar lugar a la precipitación de las fases estables e, incluso, pueden conducir al crecimiento de grandes precipitados, lo que haría perder una parte de las buenas características conseguidas por SR.

\section{ALEACIONES DE ALUMINIO PARA ALTA TEMPERATURA}

Los estudios publicados sobre estos materiales se refieren esencialmente a las aleaciones Al-Fe-X ( $\mathrm{X}=\mathrm{Ce}, \mathrm{Co}, \mathrm{Cr}, \mathrm{Mn}, \mathrm{Mo}, \mathrm{Ni}, \mathrm{Ti}, \mathrm{V}, \mathrm{Zr}, \mathrm{Y}$ y sus combinaciones) y, de forma más limitada, a las aleaciones $\mathrm{Al}-\mathrm{Cr}-\mathrm{X}(\mathrm{X}=\mathrm{Ce}, \mathrm{Co}, \mathrm{Mn}, \mathrm{Ni}, \mathrm{Si}, \mathrm{Ti}, \mathrm{V}$, $\mathrm{Zr}$ ), Al-Mn-X (X = Ce, Co, Fe, Ni, Zr) y Al-Zr-X $(\mathrm{X}=\mathrm{Cr}, \mathrm{Ti}, \mathrm{V})$ (21-23). Existen resultados sobre los materiales brutos de temple (cintas o polvos) y sobre los materiales consolidados. De un estudio sistemático llevado a cabo por Alcoa (24) en aleaciones $\mathrm{Al}-\mathrm{Fe}-\mathrm{X}(\mathrm{X}=\mathrm{Ce}, \mathrm{Mo})$ se deduce que su desarrollo industrial es posible. De hecho, las aleaciones $\mathrm{Al}-8,5 \mathrm{Fe}-2 \mathrm{Mo}$ y $\mathrm{Al}-\mathrm{Fe}-\mathrm{V}-\mathrm{Si}$ ya han sido comercializadas, si bien han encontrado problemas económicos, por lo que su producción parece estar detenida. Otro estudio de aleaciones $\mathrm{Al}-\mathrm{Cr}-\mathrm{Zr}$ (-Mn) ha sido realizado por Alcan $(3,25$ y 26). Las aleaciones $\mathrm{Al}-\mathrm{Cr}-\mathrm{Zr}$ son más fáciles de fabricar que las aleaciones de base Al-Fe y pueden endurecer durante o después de la consolidación debido a la precipitación de la fase coherente y metaestable $\mathrm{Ll}_{2}-\mathrm{Al}_{3} \mathrm{Zr}$ (27).

En un proyecto BRITE-EURAM (7) se realizó un estudio de algunos de los sistemas de aleaciones de aluminio-metal de transición para alta temperatura que en 1990 se presentaban como los más prometedores. Aunque los resultados son todavía confidenciales, ya se han hecho públicas algunas conclusiones (6). Los sistemas $\mathrm{Al}-\mathrm{Cr}(-\mathrm{Ni}, \mathrm{Mo})$ y Al-Mn-Ni resultaron excesivamente frágiles. En general, puede decirse que el níquel, a partir de 300 ${ }^{\circ} \mathrm{C}$, tiende a segregarse de la matriz, dando lugar a 
grandes precipitados de $\mathrm{Al}_{3} \mathrm{Ni}$ situados en las fronteras de grano, que fragilizan el material reduciendo la resistencia a la tracción de la aleación (28).

\section{ALEACIONES DE Al-Cr-Zr RÁPIDAMENTE SOLIDIFICADAS}

\subsection{Breve reseña histórica}

Hace años que las aleaciones $\mathrm{Al}-\mathrm{Cr}-\mathrm{Zr}$ se presentan como buenas candidatas para reemplazar a las de titanio a temperaturas entre 100 y $350{ }^{\circ} \mathrm{C}(29$ y 30$)$. Los primeros estudios sobre este material se realizaron en la Unión Soviética a partir de 1965 (31-34), seguidos por algunos trabajos en Alemania ( 35 y 36), mientras que Sahin y Jones (37) en Gran Bretaña realizaban estudios sobre la solubilidad sólida extendida, el afinamiento de grano y el endurecimiento por envejecimiento de un gran número de aleaciones Al-Zr. Desde 1984 se han realizado en Corea numerosos estudios sobre estas aleaciones (38-42).

En 1985, Midson et al. (27) y Hughes et al. (43) estudiaron el comportamiento de envejecimiento y la influencia de la velocidad de enfriamiento de las partículas de polvo sobre las propiedades mecánicas de aleaciones de base $\mathrm{Al}-\mathrm{Cr}-\mathrm{Zr}$.

En 1986 Marshall et al. (44) estudiaron el efecto del método de consolidación sobre la microestructura y las propiedades de tracción de la aleación Al4,8Cr-1,4Zr-1,4Mn. Miller et al. (45), estudiaron las propiedades de tracción de la aleación Al-5Cr$1,7 \mathrm{Zr}-1,4 \mathrm{Mn}$ a temperaturas elevadas; Saunders y Rivlin (46) estudiaron los diagramas de equilibrio de los sistemas de aleaciones Al-Cr, $\mathrm{Al}-\mathrm{Zr}$ y Al-Cr$\mathrm{Zr}$; Bendersky et al. (47) estudiaron las temperaturas de descomposición de la solución sólida y la naturaleza de las esferulitas multifase que suelen aparecer embebidas en la fase $\alpha$-Al.

En 1987 McConnel y Partridge (48 y 49) utilizando aleaciones $\mathrm{Al}-\mathrm{Cr}$ y $\mathrm{Al}-\mathrm{Cr}-\mathrm{Fe}$ templadas desde la fase vapor, estudiaron el efecto de la microestructura y de la composición sobre el módulo de Young y las propiedades de tracción. Thomas et al. (50) pusieron de manifiesto la baja densidad de las aleaciones $\mathrm{Al}-\mathrm{Cr}-\mathrm{Zr}$ comparadas con las aleaciones $\mathrm{Al}$ Fe. En estos años, Miller y Palmer (51), Palmer y Thomas (4) y Palmer et al. (52) estudiaron piezas obtenidas por extrusión y piezas forjadas, realizando un estudio muy completo de carácter preindustrial sobre las propiedades mecánicas de varias aleaciones Al-Cr-Zr obtenidas tanto por atomización como por copos y por colada de flujo laminar, y compararon su comportamiento con el de la aleación $\mathrm{Al}-8 \mathrm{Fe}$, a temperaturas de hasta $300{ }^{\circ} \mathrm{C}$.

En 1988 Park y Kim (53) estudiaron los fenómenos de envejecimiento de diversas aleaciones de este tipo; Tsakiropoulos et al. (54) estudiaron las aleaciones Al-Cr-X (X = Zr, Nb, Mo, W o Hf) y Al-Cr-Zr-X ( $\mathrm{X}=\mathrm{Mn}, \mathrm{Nb}$, Mo o $\mathrm{W}$ ) comparando los resultados con los de las aleaciones Al-Fe-Ce y Al-Fe-Mo, encontrando cierta ventaja a las aleaciones $\mathrm{Al}-\mathrm{Cr}-\mathrm{Zr}$ respecto de las $\mathrm{Al}-\mathrm{Fe}-\mathrm{Ce}$, en el sentido de que pueden ser procesadas más fácilmente y conseguir la misma dureza mediante tratamientos térmicos adecuados.

Entre 1988 y 1991, Adkins, Pan, Saunders, Shao y Tsakiropoulos (55-61) realizaron numerosos estudios sobre las condiciones de consolidación, las microestructuras y las propiedades mecánicas de estas aleaciones, estableciendo mapas de nucleación preferente de fases en función de la velocidad de enfriamiento y de la composición.

En 1990 Tezcan y Jones (62) estudiaron el efecto de la composición sobre la dureza y la respuesta al envejecimiento de polvos de Al-Cr-Zr-Mn. En 1990 y 1991 Silva, Jones, Sellars y Whiteman (63-65) estudiaron las aleaciones $\mathrm{Al}-5 \mathrm{Cr}-2 \mathrm{Zr}(-1 \mathrm{Mn})$ consolidadas mediante compactación en caliente y laminación, obteniendo buenas propiedades de resistencia a la tracción y ductilidad, mientras que Chung et al. (66) y Tu et al. (67) estudiaron las condiciones de formación de zonas sin partición de una aleación Al-Cr-Zr.

Entre 1990 y 1992 Ioannidis, Sheppard y Marshall (68-72) estudiaron los procesos de solidificación y las microestructuras en función del subenfriamiento y de la composición, así como los procesos de consolidación y la influencia de aleantes como el hierro sobre ellos. También estudiaron las propiedades mecánicas (70 y 44).

En los años 1995-96, Lieblich et al. (73-77) estudiaron la influencia del tamaño de partícula de polvo $(D)$ y las condiciones de procesado de una aleación Al-5Cr-2Zr obtenida por atomización y consolidada por extrusión en caliente, sobre las propiedades mecánicas, el endurecimiento por envejecimiento y la presión de extrusión.

Pese a su importancia, existen muy pocos estudios sobre el comportamiento en fluencia de estas aleaciones. En 1990, Mishra et al. (78) realizaron un estudio de fluencia en aleaciones $\mathrm{Al}-\mathrm{Cr}-\mathrm{Zr}$ utilizando tensiones muy bajas, con más interés teórico que práctico. Actualmente, Brahmi et al. $(18,79 \mathrm{y}$ 80 ) estudian las características de fluencia de la aleación Al-5Cr-2Zr en un amplio rango de velocidades de deformación y temperaturas de ensayo, así como la influencia del tamaño de partícula y de las variables de procesado sobre la tensión de fluencia.

\subsection{Consideraciones generales}

Las aleaciones de base $\mathrm{Al}-\mathrm{Cr}$ ofrecen ciertas ventajas frente a las de base $\mathrm{Al}-\mathrm{Fe}$, que son su alternativa, entre ellas: 
- La microestructura obtenida después de la SR depende relativamente poco de la velocidad de solidificación, en comparación con las aleaciones Al-Fe (81 y 82).

- La estabilidad térmica de la solución sólida sobresaturada de $\mathrm{Al}-\mathrm{Cr}$ es superior a la de $\mathrm{Al}-\mathrm{Fe}$. Los precipitados de $\mathrm{Al}_{13} \mathrm{Cr}_{2}$ sólo aparecen a partir de $400{ }^{\circ} \mathrm{C}$ para un mantenimiento de unas 10 h (83).

- La microestructura y la microdureza de las aleaciones Al-Cr brutas de temple se conservan prácticamente durante el tratamiento de consolidación, mientras que las de $\mathrm{Al}-\mathrm{Fe}$ se degradan en las mismas condiciones (17, 43 y 44).

- Si el procedimiento de consolidación incluye una compresión en frío, el Al-Cr bruto de temple es menos duro que el Al-Fe y se presta mejor a este tipo de operación (54).

La producción de este tipo de aleaciones plantea varios problemas. En primer lugar, hay que conocer las cantidades máximas de cromo y circonio que es posible tener en solución sólida en aluminio, en función del método de SR empleado, así como las fases estables o metaestables que aparecen en cada caso. Además, es necesario conocer la estabilidad térmica de estas fases ya que, tanto durante la consolidación de los materiales, como durante su uso, están sometidos a temperaturas elevadas. Asimismo, existen diferentes procedimientos para la consolidación, cada uno de los cuales plantea sus propios problemas. Por otra parte, es necesario conocer las propiedades mecánicas que se obtienen a $T_{\mathrm{A}}$ y a las temperaturas de servicio.

\subsection{Fases, solubilidad sólida extendida y microestructuras}

Los diagramas de equilibrio de las aleaciones binarias $\mathrm{Al}-\mathrm{Cr}$ y $\mathrm{Al}-\mathrm{Zr}$ se conocen desde antiguo (84). En ambos casos se trata de sistemas peritécticos, con campos de solubilidad sólida muy reducidos y en los que las adiciones de cromo o circonio elevan notablemente la temperatura de fusión de la aleación, lo que dificulta su preparación. También se conoce el diagrama de equilibrio ternario, al menos en su parte rica en aluminio (46), donde el líquido ternario está dominado en gran parte por la fase $\mathrm{Al}_{3} \mathrm{Zr}$. A partir del estudio de las microestructuras de solidificación de varias aleaciones peritécticas de aluminio atomizadas con gas inerte, se ha deducido un esquema del diagrama de fases $\mathrm{Al}-\mathrm{Cr}$ mostrando el campo de solubilidad sólida extendido (55, 85 y 86). La magnitud de este campo de solubilidad sólida extendido depende de la velocidad de solidificación. Así, en aleaciones Al-Cr depositadas desde la fase vapor se consigue mantener hasta un $10,7 \% \mathrm{Cr}$ en solución sólida (48 y 49), y en aleaciones con contenidos entre $2,3-6,0 \% \mathrm{Cr}$ y $2,0-5,6$ $\% \mathrm{Zr}$ obtenidas por el método de los dos pistones $\mathrm{y}$, por tanto, con velocidades de solidificación muy altas, la extensión del campo de solución sólida permite producir fenómenos de precipitación que incrementan notablemente la dureza por envejecimiento a $400-435{ }^{\circ} \mathrm{C}$, lo que pone de manifiesto una retención notable de aleantes en solución sólida (27). Este endurecimiento se atribuye principalmente a la precipitación de la fase coherente $\mathrm{Ll}_{2}-\mathrm{Al}_{3} \mathrm{Zr}$ que aparece a estas temperaturas. El cromo, después de tratada la aleación, precipita formando la fase estable $\mathrm{Al}_{13} \mathrm{Cr}_{2}$, con forma de bastones.

Marshall (87) examinó la estructura de solidificación de la aleación $\mathrm{Al}-\mathrm{Fe}-\mathrm{Cr}-\mathrm{Zr}$ atomizada con gas inerte y su comportamiento de descomposición durante el recocido del polvo. Interpretó el comportamiento de la solidificación en función del tamaño de partícula, lo que está relacionado con el subenfriamiento de nucleación. Cuando éste es muy grande, la microestructura resultante es del tipo sin partición; en caso contrario es celular. La mayor parte de las partículas contienen una microestructura mixta. Encuentra una fase(s) no identificada en las fronteras de celda del polvo rica en hierro y cromo. Durante el tratamiento térmico a $350{ }^{\circ} \mathrm{C}$ precipita la fase metaestable $\mathrm{Al}_{3} \mathrm{Zr}$, y a temperaturas mayores, las fases de equilibrio $\mathrm{Al}_{13} \mathrm{Cr}_{2}$ y $\mathrm{Al}_{13} \mathrm{Fe}_{4}$, que parecen reemplazar a la fase(s) no identificada.

Por su parte, Adkins, Saunders, Tsakiropoulos, Shao y Pan (56-60 y 86-88), en varios trabajos, desarrollaron un procedimiento, basado en la teoría de la nucleación, para predecir las fases primarias que se forman en líquidos rápidamente enfriados de las aleaciones $\mathrm{Al}-\mathrm{Cr}$ y $\mathrm{Al}-\mathrm{Zr}$ en función del tamaño de partícula, de la velocidad de extracción de calor y de la velocidad de enfriamiento en un atomizador por gas idealizado (Figs. 2a y 2b). Las conclusiones a las que llegan son las siguientes: para un diámetro de partícula dado, el subenfriamiento necesario para la formación de los intermetálicos primarios es significativamente mayor que el requerido para la formación de la solución sólida $\alpha$-Al; la formación de la solución sólida se favorece al disminuir el tamaño de partícula o aumentar la velocidad de enfriamiento; la secuencia de supresión de intermetálicos así calculada concuerda con los valores experimentales. Al aumentar la velocidad de enfriamiento o disminuir el tamaño de polvo, primero se suprime la fase $\mathrm{Al}_{11} \mathrm{Cr}_{2}$ y luego la fase $\mathrm{Al}_{13} \mathrm{Cr}_{2}$, en el caso del Al-Cr. En el caso del Al-Zr se puede suprimir la fase $\mathrm{Al}_{3} \mathrm{Zr}$. Basándose en la teoría de la nucleación calcularon los tiempos de incubación de las distintas fases del sistema $\mathrm{Al}-\mathrm{Cr}$, encontrando que está controlado por el subenfriamiento y por la 

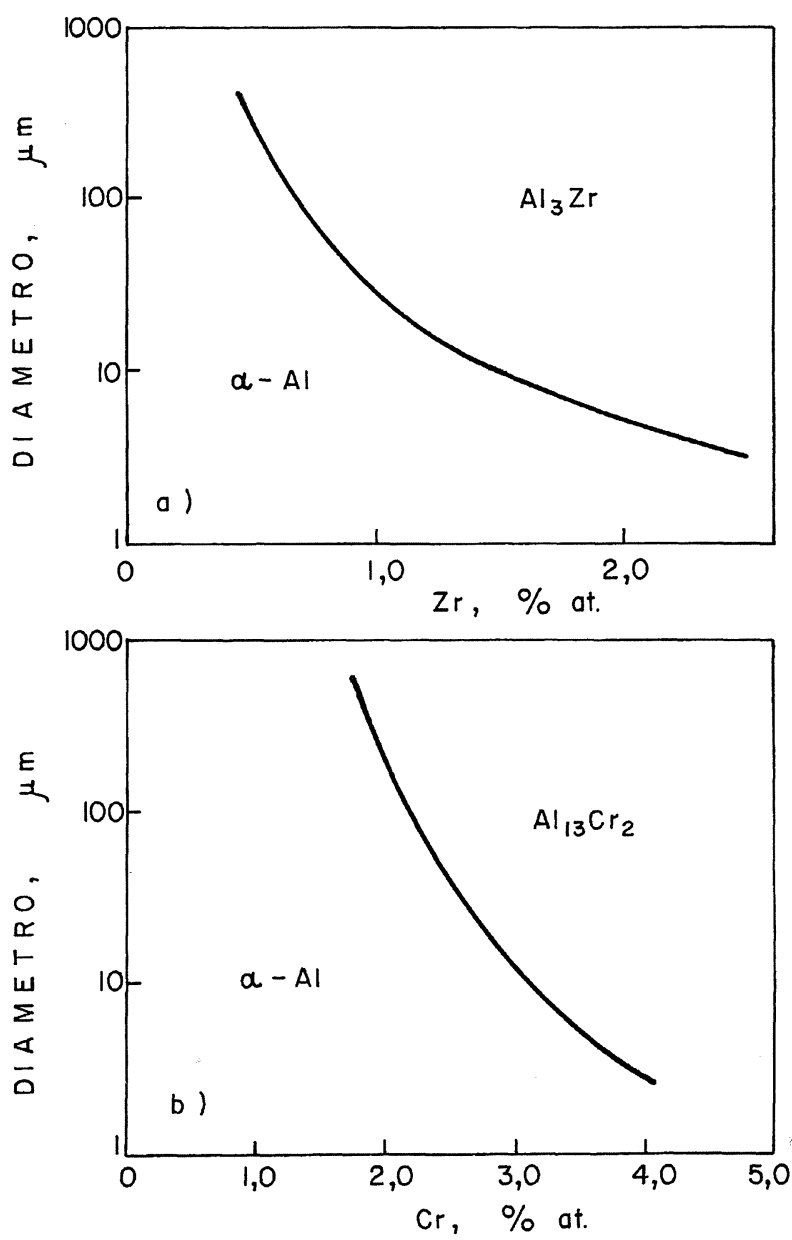

FIG. 2.- Mapa de predicción de fases predominantes en las aleaciones: a) $\mathrm{Al}-\mathrm{Zr}$; b) $\mathrm{Al}-\mathrm{Cr}$.

FIG. 2.- Predominant nucleation map predicted phases in the alloys: a) Al-Zr; b) Al-Cr alloys.

difusividad en el caldo. También calcularon el subenfriamiento, la velocidad de enfriamiento y el tamaño de partícula críticos para la selección de una fase primaria.

Bendersky et al. (47) estudiaron el comportamiento de la microestructura y la descomposición de la solución sólida de aleaciones Al-Cr solidificadas rápidamente por el método del temple sobre rueda, con contenidos de hasta un $15 \% \mathrm{Cr}$. En las aleaciones con 1 y $2 \% \mathrm{Cr}$ aparece únicamente la fase $\alpha-\mathrm{Al}$, solución sólida sobresaturada, lo que indica que para estas velocidades de enfriamiento es posible mantener en solución sólida un mínimo de $2 \% \mathrm{Cr}$. Sin embargo, para la aleación con $5 \% \mathrm{Cr}$, hay ya una microestructura de transición. En algunas regiones, esta microestructura es de granos de $\alpha-\mathrm{Al}$ sobresaturada de cromo, pero en otras hay esferulitas multifase embebidas en la matriz de aluminio. La cantidad de esferulitas aumenta al aumentar la cantidad de cromo en la aleación. Estas esferulitas están formadas por un corazón o núcleo que contiene un $22 \%$
Cr, formado por dos o más fases intermetálicas, y que está rodeado de cristales en forma de pétalos de $\mathrm{Al}_{13} \mathrm{Cr}_{2}$, cuya concentración disminuye hasta el $5 \%$ Cr. Asimismo, determinaron las temperaturas de descomposición de la solución sólida en función de la concentración de cromo.

Los tratamientos térmicos producen la segregación de soluto en fronteras de grano y, a temperaturas más altas, la precipitación en la matriz (Fig. 3). Ioannidis y Sheppard (68) estudiaron los procesos de solidificación que tienen lugar durante la atomización con gas inerte de las aleaciones $\mathrm{Al}-4 \mathrm{Cr}-1 \mathrm{Fe}$, Al-6,43Cr-1,67Zr y Al-5Cr-2Zr, así como las microestructuras resultantes. En partículas pequeñas, el gran subenfriamiento de nucleación alcanzado da lugar a la solidificación sin partición en las aleaciones con bajo contenido de cromo, y a una estructura globular en las de alto contenido de cromo. En las partículas de tamaño medio se presenta una morfología mixta, dependiendo del subenfriamiento de nucleación alcanzado y de la velocidad de extracción de calor al gas. A medida qué aumenta el tamaño de las partículas, la aleación con menor contenido de cromo muestra, sobre todo, morfologías celulares y, al aumentar el contenido de cromo, van apareciendo glóbulos de fases metaestables ricas en este elemento y/o se forman agregados de partículas individuales. La difracción de rayos $\mathrm{X}$ indica la presencia de fases no identificadas, probablemente formadas directamente en la gota líquida. En definitiva, la posibilidad de conseguir solución sólida depende de la cantidad de aleante presente y de la velocidad de solidificación.

Un estudio del efecto de la velocidad de solidificación en las aleaciones $\mathrm{Al}-5,1 \mathrm{Cr}-1,4 \mathrm{Zr}-1,5 \mathrm{Mn}$ y

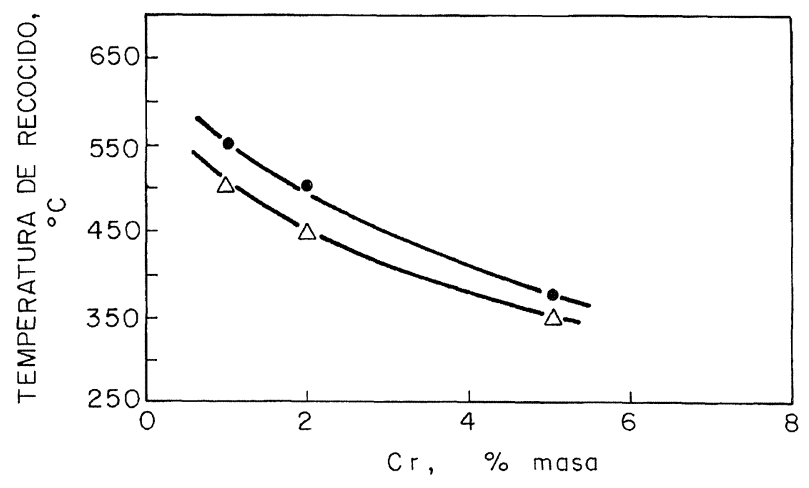

FIG. 3.- Diagrama temperatura de recocido-composición, donde las curvas representan el inicio de la precipitación $(\bullet)$ en las fronteras de grano $(\mathrm{PFG})$ y $(\Delta)$ en el interior de los granos para recocidos de $6 \mathrm{~h}$.

FIG. 3.- Annealing temperature-composition diagram, where curves represent the beginning of precipitation $(\bullet)$ on grain boundaries $(G B P)$ and $(\Delta)$ inside the grains for $6 \mathrm{~h}$ anneals. 
Al-3,3Cr-0,7Zr-0,7Mn, mediante solidificación en una coquilla en forma de cuña, permitió conocer las microestructuras y las fases que aparecen para una serie continua de velocidades de solidificación, confirmando que, en este tipo de aleaciones peritécticas, un subenfriamiento de nucleación alto favorece la nucleación de la fase $\alpha-\mathrm{Al}$ frente a la de los compuestos intermetálicos (59). En la aleación con 5,1 $\% \mathrm{Cr}$, la fase $\mathrm{Al}_{11} \mathrm{Cr}_{2}$ desaparece para velocidades de enfriamiento de $5 \times 10^{2}{ }^{\circ} \mathrm{C} \cdot \mathrm{s}^{-1}$ y la fase $\mathrm{Al}_{13} \mathrm{Cr}_{2}$ lo hace a $10^{4}{ }^{\circ} \mathrm{C} \cdot \mathrm{s}^{-1}$.

En la aleación con 3,3\% Cr, la formación de una estructura celular libre de intermetálicos tiene lugar a velocidades entre $5 \times 10^{2}$ y $2 \times 10^{3}{ }^{\circ} \mathrm{C} \cdot \mathrm{s}^{-1}$. Además, esta aleación muestra un considerable endurecimiento al tratarla a $400{ }^{\circ} \mathrm{C}$ durante $25 \mathrm{~h}$, lo que indica que gran parte del circonio ha permanecido en solución sólida. Obteniendo las mismas aleaciones (60) por atomización con helio, las microestructuras de los polvos presentan una secuencia que depende de su tamaño o, lo que es lo mismo, de su velocidad de enfriamiento. Esta secuencia, empezando por las partículas más pequeñas, es la siguiente: a) microestructura libre de segregación, b) celular $\alpha$-Al, c) celular $\alpha$-Al+precipitados esféri$\cos$ ricos en $\mathrm{Cr}$ y $\mathrm{Mn}$, d) celular $\alpha$-Al+intermetálicos primarios de $\mathrm{Al}_{13} \mathrm{Cr}_{2}$.

Las microestructuras de SR están influidas por el coeficiente de transferencia de calor y por el modelo de solidificación. Basándose en los mapas de nucleación (56 y 57) se puede conocer el tamaño de partícula para el que se obtienen las microestructuras deseadas. Éstas dependen de la velocidad del frente de solidificación que, a su vez, está controlado por la velocidad de extracción de calor al gas y por el subenfriamiento inicial. Para partículas muy pequeñas $(D<1 \mu \mathrm{m})$, sin partición, la solidificación tiene lugar con frentes cuya velocidad supera la velocidad de estabilidad absoluta. Para partículas mayores $(D \sim 5-10 \mu \mathrm{m})$, la presencia de una zona con dos microestructuras distintas parece que se debe a la disminución de la velocidad del frente de solidificación durante la recalescencia.

El estudio de la formación de zonas sin partición en la aleación Al-5,75Cr-1,96Zr, obtenida por atomización con gas inerte, permitió relacionar el aspecto superficial con la velocidad de enfriamiento (67). Una superficie lisa está relacionada con una velocidad de enfriamiento alta. Para estas condiciones de enfriamiento aparecen tres tipos de microestructuras: a) sin partición, b) microcelular y c) celular-dendrítica. También se estableció una distinción entre el aspecto sin partición observado por microscopía óptica y electrónica de barrido y la auténtica ausencia de micropartición observada por microscopía electrónica de transmisión, que requiere, para el cromo y el circonio, velocidades de enfriamiento altísimas, del orden de $10^{12}{ }^{\circ} \mathrm{C} \cdot \mathrm{s}^{-1}$. La estructura sin partición aparente se encuentra en partículas muy pequeñas $(\sim 10 \mu \mathrm{m})$ y también en partículas que han solidificado por choque con otras ya sólidas. En este caso, cuando un filamento grande con un cierto grado de subenfriamiento choca con otra partícula ya solidificada, la extracción de calor pasa de ser por convección (atomización) a ser por conducción (mucho más rápida), con lo que se consigue casi instantáneamente un gran subenfriamiento que facilita la nucleación heterogénea en la superficie de contacto. Entonces, la intercara sólido-líquido se desplaza rápidamente de forma laminar dando lugar a una estructura sin partición. Claro está que puede haber zonas del filamento alejadas de los puntos de impacto, a las que la intercara sólido-líquido llega con menor velocidad a causa del calor latente generado durante la recalescencia. Entonces, esta intercara se inestabiliza y da lugar a una estructura microcelular.

Cuando se comparan los efectos sobre la microestructura de la atomización con gas y la colada de flujo laminar (72) de la aleación Al-5Cr-2Zr se encuentra qué las cintas tienen mayor homogeneidad que los polvos, se eliminan los precipitados de intermetálicos mayores de $0,5 \mu \mathrm{m}$ y se retiene más aleante en solución sólida. En un estudio de la distribución de nucleantes heterogéneos en polvos de Al-Cr (58) se analizó la distribución de intermetálicos en polvos de distintos tamaños atomizados con gas inerte de la aleación $\mathrm{Al}-5 \mathrm{Cr}$, y se desarrolló un método para calcular la distribución de intermetálicos en función del tamaño del polvo y de la fracción de volumen de precipitados, mostrando que sólo en los polvos muy pequeños se encuentran estructuras celulares libres de intermetálicos.

Sánchez et al. (89), trabajando con una aleación de $\mathrm{Al}-5,7 \mathrm{Cr}-1 \mathrm{Mo}$, solidificada rápidamente por atomización centrífuga en helio, con una velocidad relativa gota/gas estimada en $60 \mathrm{~ms}^{-1}$, encontraron tres tipos de microestructuras: celular, globular y en forma de rosetas lo que les llevó a establecer un mapa de nucleación preferente en función del tamaño de la gota y del contenido en cromo de la aleación.

En resumen, en las aleaciones rápidamente solidificadas $\mathrm{Al}-\mathrm{Cr}-\mathrm{Zr}$, las fases estables o metaestables que aparecen y las cantidades de cromo o circonio retenidas en solución sólida dependen de la velocidad de enfriamiento y del contenido de aleantes. Para velocidades muy altas como, por ejemplo, el temple desde fase vapor, se puede llegar a retener en solución sólida hasta un $10 \% \mathrm{Cr}$. Utilizando métodos de SR más económicos, como la colada de flujo laminar, CFL, o la atomización con gas, la cantidad de cromo retenido en solución sólida depende de su concentración en la aleación y de la velocidad de enfriamiento; es decir, no sólo es distinta para los diferentes métodos de solidificación, 
sino también para cada gota solidificada, dependiendo de su tamaño. En general, la secuencia suele ser la siguiente: para partículas muy pequeñas (subenfriamiento de nucleación muy grande) se produce una gran velocidad de solidificación y se obtiene una microestructura sin partición; para partículas un poco más grandes, el calor producido durante la recalescencia frena el frente de solidificación y aparece una estructura mixta sin partición y con regiones celulares. Para subenfriamientos menores puede tenerse, en principio, una estructura celular, pero el calor de la recalescencia puede dar lugar a la precipitación de la fase $\mathrm{Al}_{13} \mathrm{Cr}_{2}$; para partículas mayores se favorece la formación de la fase $\mathrm{Al}_{13} \mathrm{Cr}_{2}$, que luego es embebida por la matriz. Por último, con subenfriamientos bajos, los intermetálicos tienen tiempo de crecer formando grandes dendritas antes de que solidifique el resto del aluminio. El circonio sigue una secuencia semejante, precipitando primero la fase coherente metaestable $\mathrm{L1}_{2}-$ $\mathrm{Al}_{3} \mathrm{Zr}$, que a temperaturas más altas se transforma en la fase tetragonal, estable e incoherente $\mathrm{DO}_{23}$ $\mathrm{Al}_{3} \mathrm{Zr}(84)$.

\subsection{Estabilidad térmica y consolidación}

La consolidación de los polvos, cintas o copos requiere la aplicación de procesos como la extrusión o la laminación. Ello significa que estos productos, que se encuentran parcialmente en estado metaestable, tienen que ser sometidos a procesos termomecánicos que pueden dar lugar a fenómenos de precipitación en la solución sólida sobresaturada, a crecimiento de los intermetálicos primarios, a transformación de fases, a crecimiento de grano o de subgrano, etc. Todos, o alguno de estos fenómenos, puede conducir a la pérdida de las buenas propiedades conseguidas con la SR.

Las aleaciones de aluminio de forja, obtenidas por SR, tienen mejores propiedades de resistencia mecánica, rigidez y estabilidad térmica que las obtenidas por métodos tradicionales (90). Entre ellas, las aleaciones $\mathrm{Al}-\mathrm{Cr}$ y $\mathrm{Al}-\mathrm{Zr}$ son especialmente adecuadas para ampliar el campo de solubilidad en estado sólido y estabilizarlo, por su elevada resistencia a la descomposición de la solución sólida. Además, en el caso del circonio, se consigue un notable endurecimiento por envejecimiento a temperaturas altas. Midson et al. (27) estudiaron la estabilidad térmica de las aleaciones rápidamente solidificadas Al-Cr-Zr, con contenidos entre 2,3-6,0 $\% \mathrm{Cr}$ y $2,0-5,6 \% \mathrm{Zr}$, poniendo de manifiesto que los dos aleantes en solución sólida interaccionan entre sí mejorando las propiedades de las aleaciones binarias.

El estudio del envejecimiento muestra que el endurecimiento después de $1 \mathrm{~h}$ a $400-435^{\circ} \mathrm{C}$ se debe fundamentalmente a la precipitación de la fase $\mathrm{L1}_{2}-\mathrm{Al}_{3} \mathrm{Zr}$ aunque, en ausencia de cromo, esta fase sólo aparece a temperaturas más altas. $\mathrm{La}$ fase $\mathrm{L1}_{2-}$ $\mathrm{Al}_{3} \mathrm{Zr}$ precipita en forma de abanico. El cromo precipita en forma de bastones de $\mathrm{Al}_{13} \mathrm{Cr}_{2}$, pero no participa en el endurecimiento por envejecimiento. $\mathrm{La}$ adición de circonio a las aleaciones binarias $\mathrm{Al}-\mathrm{Cr}$ puede rebajar hasta $70{ }^{\circ} \mathrm{C}$ la temperatura de descomposición de la solución sólida sobresaturada, definida como la temperatura a la que se pierde la mitad del contenido de aleante en solución al cabo de $1 \mathrm{~h}$ de tratamiento. En cualquier caso, esta temperatura de descomposición se encuentra entre 450 y $500{ }^{\circ} \mathrm{C}$.

Un estudio de Hughes et al. (43) sobre la estabilidad térmica de copos de Al-Cr- $\mathrm{Zr}$ con contenidos entre 0,5-5,5 Cr y 0,25-2,5\% Zr, comparada con la de polvos de igual composición atomizados con aire, indica que a temperaturas entre 350 y $450{ }^{\circ} \mathrm{C}$ el cromo aumenta la estabilidad térmica de estos polvos, y el circonio aporta el endurecimiento por envejecimiento, dependiendo su efecto de la composición de la aleación. En ningún caso se produce precipitación de compuestos ternarios. Los copos de la aleación Al-3Cr-1Zr pasan de una dureza de $55 \mathrm{HV}$, en estado de recién templados, a $125 \mathrm{HV}$ después de tratados $1 \mathrm{~h}$ a $450{ }^{\circ} \mathrm{C}$, mientras que los de la aleación Al-3Cr-3Zr pasan de 75 HV a 190 $\mathrm{HV}$. El análisis térmico diferencial muestra que en los copos el endurecimiento por envejecimiento se debe a dos reacciones de descomposición que tienen su máximo a 400 y a $500{ }^{\circ} \mathrm{C}$, causadas por la precipitación de la fase metaestable $\mathrm{Al}_{3} \mathrm{Zr}$ y de la fase estable $\mathrm{Al}_{13} \mathrm{Cr}_{2}$, respectivamente.

La extrusión en caliente, que es el método más utilizado de consolidación de polvos, requiere precalentar los contenedores de los mismos durante bastante tiempo hasta alcanzar la temperatura de extrusión, lo que conduce a un crecimiento de los precipitados, perjudicando así las propiedades del material consolidado. Por ello, se ha intentado emplear otros métodos de consolidación. Marshall et al. (44) compararon los efectos sobre el posterior endurecimiento por envejecimiento de barras obtenidas por extrusión hidrostática y por extrusión en caliente de diversas aleaciones Al-Cr-Zr-Mn atomizadas con aire. En ambos casos, los polvos se compactaron en frío hasta una densidad aparente del $75 \%$, y luego en caliente hasta el $100 \%$. La extrusión hidrostática, que no requiere precalentamiento más que durante la precompactación, minimiza el tiempo de exposición a la máxima temperatura alcanzada durante la extrusión, mientras que en la extrusión en caliente la barra sufre el calentamiento propio de las fricciones de la extrusión. Las mejores propiedades mecánicas corresponden a las aleaciones obtenidas por extrusión hidrostática, lo que confirma que hay cierta inestabilidad de las fases 
presentes frente a la temperatura, posiblemente precipitación y crecimiento de la fase $\mathrm{Al}_{13} \mathrm{Cr}_{2}$.

Ioannidis et al. (69) extruyeron las aleaciones Al-5Cr-2Zr, Al-6,43Cr-1,67Zr y Al-4Cr-1Fe utilizando un método de calentamiento rápido del tocho mediante un horno de inducción. El proceso consiste en una compactación en frío hasta conseguir una densidad aparente del $87 \%$ de la teórica, seguida de un calentamiento rápido y extrusión en caliente, aunque también se consiguen densidades del $94 \%$ utilizando un método de compactación de doble pistón (70). Las presiones de extrusión dependen fundamentalmente de la composición de la aleación, así como de la velocidad del pistón, de la temperatura y de la relación de extrusión (76). La consolidación de la aleación con hierro es más fácil que la de las que contienen circonio, ya que este elemento aumenta la presión de extrusión. El método de calentamiento rápido por inducción requiere que el material tenga poca porosidad, por lo que es necesario realizar una precompactación en frío. Siguiendo con métodos de calentamiento rápido semejantes, Marshall et al. (72) estudiaron el efecto de la extrusión directa e indirecta sobre las condiciones de extrusión de la aleación Al-5Cr-2Zr, obtenida por atomización o por colada de flujo laminar. La extrusión indirecta, a causa de su menor fricción con el contenedor, reduce el pico de extrusión, lo que permite bajar la temperatura de extrusión en al menos $25^{\circ} \mathrm{C}$. Durante el precalentamiento, pese a su corta duración, la aleación endurece por precipitación de la fase metaestable $\mathrm{Al}_{3} \mathrm{Zr}$.

Lieblich et al. (73) examinaron el efecto del tamaño de partícula, $D$, sobre la dureza y las propiedades de tracción en la aleación $\mathrm{Al}-5 \mathrm{Cr}-2 \mathrm{Zr}$ atomizada con argón. Las condiciones de consolidación, especialmente la temperatura de extrusión $\left(T_{\text {ext }}\right)$, son muy importantes. Así, el aumento de la $T_{\text {ext }}$ desde 300 a $450{ }^{\circ} \mathrm{C}$, aunque reduce un $40 \%$ la presión de extrusión, da lugar a peores propiedades mecánicas. Un descenso de la velocidad del pistón o de la relación de extrusión (RE) no tiene influencia sobre la dureza y la resistencia, y por lo tanto sobre la presión de extrusión, por lo que es posible consolidar el material a temperaturas tan bajas como $300{ }^{\circ} \mathrm{C}$. Se consiguen altos niveles de dureza y resistencia combinando un tamaño de partícula pequeño con una temperatura de extrusión baja $\left(D<25 \mu \mathrm{m}\right.$ y $\left.T_{\text {ext }}=350{ }^{\circ} \mathrm{C}\right)$. Esta influencia del $D$ y de la $T_{\text {ext }}$ es confirmada por Caruana et al. (75) al estudiar su efecto sobre la dureza de la barras recién extruídas y después de un tratamiento térmico. Por el contrario, el sobrecalentamiento del caldo antes de la atomización no tiene efecto sobre la presión de extrusión; la velocidad del pistón y la RE afectan poco, si bien a bajas velocidades del pistón desaparece el pico de extrusión (76).
En resumen, parece que la extrusión en caliente es el método ideal para consolidar este tipo de materiales. La extrusión debe realizarse a temperaturas lo más bajas posible, para no alterar la microestructura de SR, aunque siempre se produce precipitación de la fase metaestable $\mathrm{Al}_{3} \mathrm{Zr}$. Además, el precalentamiento debe realizarse durante el menor tiempo posible, aun cuando esto pueda impedir la desgasificación de los polvos. La velocidad de extrusión debe ser baja, para evitar una subida excesiva de la presión de extrusión, limitada por la capacidad de las prensas.

\subsection{Propiedades mecánicas}

En la tabla I se resumen algunas de las propiedades de tracción a $T_{\mathrm{A}}$ y a temperaturas elevadas encontradas en la literatura para algunas aleaciones $\mathrm{Al}-\mathrm{Cr}-\mathrm{Zr}$ y otras similares obtenidas por SR.

La importancia de estas aleaciones está fundada en sus posibles aplicaciones a temperaturas elevadas, por lo que no sólo deben estudiarse sus propiedades a $T_{\mathrm{A}}$, en función de los métodos de solidificación y de consolidación empleados, sino sus propiedades a temperaturas elevadas. Así, por ejemplo, la aleación Al-5Cr-1,5Zr-1,0Mn atomizada en aire, compactada en frío y laminada, presenta distintas propiedades mecánicas en función de la velocidad de enfriamiento sufrida por cada tamaño de partícula de polvo (43).

Para una velocidad de enfriamiento de $2,5 \times 10^{4}$ ${ }^{\circ} \mathrm{C} \cdot \mathrm{s}^{-1}(D<75 \mu \mathrm{m})$ se alcanza un límite elástico convencional al $0,2 \%$ de deformación $(Y S)$ de 530 $\mathrm{MPa}$, mientras que para una velocidad de 1,6 $\times 10^{2}$ ${ }^{\circ} \mathrm{C} \cdot \mathrm{s}^{-1}(D>420 \mu \mathrm{m})$ sólo se alcanzan $396 \mathrm{MPa}$. En promedio, puede considerarse que las propiedades mecánicas toman los siguientes valores: $Y S=500$ $\mathrm{MPa}$; carga de rotura, $U T S=550 \mathrm{MPa}$; y alargamiento a rotura, $\varepsilon_{\mathrm{R}}=6 \%$. Sin embargo, cuando el material se trata $100 \mathrm{~h}$ a $300^{\circ} \mathrm{C}$ y se ensaya a esta misma temperatura, el valor de la UTS baja hasta $230 \mathrm{MPa}$.

Con una aleación muy parecida a la anterior, Al$4,8 \mathrm{Cr}-1,4 \mathrm{Zr}-1,4 \mathrm{Mn}$, se realizaron estudios comparativos de las propiedades mecánicas obtenidas cuando la consolidación se realiza por extrusión hidrostática o en caliente (Tabla I) (44). Después del mismo tratamiento térmico, las propiedades mecánicas de las barras extruídas hidrostáticamente experimentan una ligera mejoría, mientras que las de la extrusión convencional disminuyen cuando se ensayan a $T_{\mathrm{A}}$. Los valores obtenidos para un procedimiento de consolidación y otro son, respectivamente: $Y S=505$ y $293 \mathrm{MPa} ; U T S=527$ y 317 $\mathrm{MPa}$, y $\varepsilon_{\mathrm{R}}=15,0$ y $13,5 \%$. Para la extrusión hidrostática se encontró una tenacidad $K_{\mathrm{Q}}=14,4$ $\mathrm{MPa} \mathrm{m}^{1 / 2}$. 
TABLA I.- Propiedades mecánicas

TABLE I.- Mechanical properties

\begin{tabular}{|c|c|c|c|c|c|c|c|c|c|c|}
\hline & & & \multicolumn{3}{|c|}{ Temp. ambiente } & \multicolumn{4}{|c|}{ Alta temperatura } & \\
\hline $\begin{array}{l}\text { Aleación Al- } \\
\% \text { en masa }\end{array}$ & Obtención & Consolidación & $\begin{array}{l}Y S \\
\text { MPa }\end{array}$ & $\begin{array}{l}U T S \\
\mathrm{MPa}\end{array}$ & $\begin{array}{l}\varepsilon_{\mathrm{R}} \\
\%\end{array}$ & $\begin{array}{l}Y S, \\
\mathrm{MPa}\end{array}$ & $\begin{array}{l}U T S, \\
\mathrm{MPa}\end{array}$ & $\begin{array}{l}\varepsilon_{\mathrm{R}} \\
\%\end{array}$ & $\begin{array}{l}\mathrm{T}, \\
{ }^{\circ} \mathrm{C}\end{array}$ & Ref. \\
\hline $5 \mathrm{Cr}-1,5 \mathrm{Zr}-1 \mathrm{Mn}$ & $\begin{array}{l}\text { Atomizac. } \\
2,5 \cdot 10^{4}{ }^{\circ} \mathrm{C} / \mathrm{s} \\
1,6 \times 10^{2}{ }^{\circ} \mathrm{C} / \mathrm{s}\end{array}$ & Lamin. & $\begin{array}{l}530 \\
396\end{array}$ & & $\begin{array}{r}6 \\
10\end{array}$ & & 230 & & 300 & 43 \\
\hline $4,8 \mathrm{Cr}-1,4 \mathrm{Zr}-1,4 \mathrm{Mn}$ & Atomizac. & $\begin{array}{l}\text { Ext. en caliente } \\
\text { Hidrost. }\end{array}$ & $\begin{array}{l}315 \\
484\end{array}$ & $\begin{array}{l}349 \\
515\end{array}$ & $\begin{array}{l}7,5 \\
8\end{array}$ & & & & & 44 \\
\hline $\begin{array}{l}5 \mathrm{Cr}-2 \mathrm{Zr} \\
3 \mathrm{Cr}-3 \mathrm{Zr} \\
\end{array}$ & $\begin{array}{l}\text { Atomizac. } \\
\text { CFL }\end{array}$ & Ext. $400{ }^{\circ} \mathrm{C}$ & $\begin{array}{l}478 \\
468 \\
\end{array}$ & $\begin{array}{l}498 \\
487\end{array}$ & $\begin{array}{r}10,5 \\
5,8 \\
\end{array}$ & 250 & 290 & & 300 & 52 \\
\hline $\begin{array}{l}5,2 \mathrm{Cr}-1,4 \mathrm{Zr}-1,3 \mathrm{Mn} \\
3,5 \mathrm{Cr}-0,5 \mathrm{Zr}-1,0 \mathrm{Mn}\end{array}$ & Atomizac. & Extrusión & $\begin{array}{l}512 \\
384\end{array}$ & $\begin{array}{l}543 \\
414\end{array}$ & $\begin{array}{l}5,6 \\
6,6\end{array}$ & & & & & 61 \\
\hline $5 \mathrm{Cr}-2 \mathrm{Zr}-1 \mathrm{Mn}$ & $\begin{array}{l}\text { Atomizac. } \\
\text { CFL }\end{array}$ & Extrusión & & $\begin{array}{l}510 \\
540\end{array}$ & $\begin{array}{r}14 \\
9\end{array}$ & & $\begin{array}{l}275 \\
310\end{array}$ & $\begin{array}{l}6 \\
11\end{array}$ & 316 & 65 \\
\hline $\begin{array}{l}4 \mathrm{Cr}-1 \mathrm{Fe} \\
6,43 \mathrm{Cr}-1,67 \mathrm{Zr} \\
5 \mathrm{Cr}-2 \mathrm{Zr}\end{array}$ & Atomizac. & $\begin{array}{l}\text { Extrusión } \\
450^{\circ} \mathrm{C}\end{array}$ & $\begin{array}{l}275 \\
370 \\
387\end{array}$ & $\begin{array}{r}325 \\
375 \\
389\end{array}$ & $\begin{array}{l}19 \\
20 \\
22\end{array}$ & & & & & 69 \\
\hline $\begin{array}{l}4 \mathrm{Cr}-1 \mathrm{Fe} \\
5 \mathrm{Cr}-2 \mathrm{Zr} \\
6,6 \mathrm{Cr}-1,7 \mathrm{Zr}\end{array}$ & Atomizac. & $\begin{array}{l}\text { Extrusión } \\
450^{\circ} \mathrm{C}\end{array}$ & $\begin{array}{l}260 \\
396 \\
363\end{array}$ & $\begin{array}{l}310 \\
407 \\
369\end{array}$ & $\begin{array}{l}20,1 \\
17,9 \\
17,2\end{array}$ & & & & & 70 \\
\hline $5 \mathrm{Cr}-2 \mathrm{Zr}$ & $\begin{array}{l}\text { Atomizac. } \\
\text { CFL } \\
\text { Atomizac. } \\
\text { CFL }\end{array}$ & $\begin{array}{l}\text { Ext-directa } \\
450{ }^{\circ} \mathrm{C} \\
\text { Ext-indirecta } \\
450{ }^{\circ} \mathrm{C}\end{array}$ & $\begin{array}{l}390 \\
400 \\
345 \\
350\end{array}$ & $\begin{array}{l}400 \\
415 \\
350 \\
387\end{array}$ & $\begin{array}{l}17 \\
19 \\
18 \\
20\end{array}$ & $\begin{array}{l}121 \\
117\end{array}$ & $\begin{array}{l}131 \\
127\end{array}$ & $\begin{array}{l}1 \\
5\end{array}$ & Alta & 72 \\
\hline $5 \mathrm{Cr}-2 \mathrm{Zr}$ & Atomizac. & $\begin{array}{l}\text { Extrusión } \\
350^{\circ} \mathrm{C}\end{array}$ & 383 & 391 & 11,9 & & & & & 73 \\
\hline
\end{tabular}

De todo ello se deduce que la extrusión hidrostática da mejores resultados que la extrusión en caliente, lo que es lógico, dado que cualquier tratamiento térmico, como el precalentamiento empleado en la extrusión en caliente produce la precipitación de la fase metaestable $\mathrm{Al}_{3} \mathrm{Zr}$. Palmer et al. (52) llevan a cabo un estudio muy completo de carácter preindustrial en aleaciones pulvimetalúrgicas Al$\mathrm{Cr}-\mathrm{Zr}$, realizando comparaciones con las aleaciones Al-Fe-X. La extrusión se desarrolló a dos escalas: una escala de laboratorio y una semicomercial. De los ensayos de tracción a $T_{\mathrm{A}}$ se tiene que las mejores propiedades mecánicas se obtienen por el material extruído a la $T_{\text {ext }}=425^{\circ} \mathrm{C}$, con los valores de la $U T S=490 \mathrm{MPa}$, y del $\varepsilon_{\mathrm{R}}=10-12 \%$. En ensayos de tracción a $T_{\mathrm{A}}$ llevados a cabo en las extrusiones semicomerciales $\left(T_{\text {ext }}=400{ }^{\circ} \mathrm{C}\right)$ de las aleaciones Al-5Cr-2Zr y Al-3Cr-3Zr, se encontraron los siguientes valores: $Y S=478$ y $468 \mathrm{MPa}, U T S=498$ y $487 \mathrm{MPa}$ y $\varepsilon_{\mathrm{R}}=10,5$ y $5,8 \%$, respectivamente. El módulo de Young alcanza 80 ó $90 \mathrm{GPa}$. A la temperatura de ensayo de $300{ }^{\circ} \mathrm{C}$ se tienen los valores: $Y S$ $\sim 250 \mathrm{MPa}$ y $U T S \sim 290 \mathrm{MPa}$.
Una forma de mejorar notablemente las propiedades de estas aleaciones es recurrir al temple desde la fase vapor, donde las velocidades de deposición son muy altas, ya que no se puede hablar de solidificación. De este modo, McConnell y Partridge (48 y 49) han preparado aleaciones $\mathrm{Al}-\mathrm{Cr}$ y $\mathrm{Al}-$ $\mathrm{Cr}-\mathrm{Fe}$ con contenidos de hasta $11,7 \mathrm{Cr}$ y $1,88 \% \mathrm{Fe}$, y han estudiado su efecto sobre el módulo de Young y las propiedades de tracción. El módulo aumenta $5,7 \mathrm{GPa}$ por cada $1,91 \% \mathrm{Cr}(1$ at \%) en solución sólida, alcanzando $96 \mathrm{GPa}$. La transformación del cromo de la solución sólida en los precipitados $\mathrm{Al}_{13} \mathrm{Cr}_{2}$ y las adiciones de hierro no afectan al módulo de Young. Por cada 1,91 $\mathrm{Cr}$ en solución sólida, el $Y S$ aumenta en 44,7 MPa. En presencia de cromo en solución, el hierro está en forma de precipitados de 3-5 nm de diámetro y aumenta el $Y S$ en $333 \mathrm{MPa}$ por cada $2,04 \% \mathrm{Fe}$ ( 1 at. $\%$ ).

El trabajado y recocido a $260-300{ }^{\circ} \mathrm{C}$ de estos materiales no afecta a la microestructura ni al $Y S$. Las aleaciones $\mathrm{Al}-\mathrm{Cr}$-Fe llegan a alcanzar valores del $Y S$ de $818 \mathrm{MPa}$ en condiciones muy específicas. En el valor del $Y S$ intervienen varios factores: a) la 
solución sólida aporta un $25 \%$, b) los precipitados ricos en hierro un $42 \%$, y c) la densidad de dislocaciones y el tamaño de celda un $33 \%$. En el caso de las aleaciones $\mathrm{Al}-\mathrm{Cr}$, al valor del $Y S$ contribuyen: a) la solución sólida con el $40 \%$ y b) la densidad de dislocaciones y el tamaño de celda con el $60 \%$. Para fracciones de volumen de precipitados entre 0,1-0,4, el $Y S$ aumenta al aumentar la fracción de volumen y al disminuir el tamaño de grano y el tamaño de partícula. La resistencia de las aleaciones Al-Cr es máxima cuando todo el cromo está retenido en solución sólida. Puesto que sólo las aleaciones obtenidas por temple, a partir de la fase vapor, retienen todo el cromo en solución sólida para altos contenidos de cromo y después del trabajado, esta técnica es la más eficiente para emplear el cromo como elemento aleante. Sin embargo, desde el punto de vista industrial, esta técnica no parece muy práctica.

Adkins et al. (61) estudiaron las propiedades mecánicas en las aleaciones $\mathrm{Al}-5,2 \mathrm{Cr}-1,4 \mathrm{Zr}-1,3 \mathrm{Mn}$ y Al-3,5Cr-0,5Zr- $1 \mathrm{Mn}$ obtenidas por atomización y extruídas en caliente. A $T_{\mathrm{A}}$ alcanzan los siguientes valores: $U T S=543$ y $414 \mathrm{MPa}, Y S=512$ y 384 $\mathrm{MPa}$, y $\varepsilon_{\mathrm{R}}=5,6$ y $6,6 \%$, respectivamente. Estos valores se aproximan a la línea de objetivos indicada en la figura 1. Ioannidis y Sheppard (69), en ensayos de tracción a $T_{\mathrm{A}}$ realizados en la dirección longitudinal en las aleaciones $\mathrm{Al}-5 \mathrm{Cr}-2 \mathrm{Zr}, \mathrm{Al}-$ $6,43 \mathrm{Cr}-1,67 \mathrm{Zr}$ y $\mathrm{Al}-4 \mathrm{Cr}-1 \mathrm{Fe}$, obtenidas por atomización, compactación en frío y extrusión a $450{ }^{\circ} \mathrm{C}$, alcanzan los siguientes valores: $Y S=387,370$ y $275 \mathrm{MPa} ; U T S=389,375$ y $325 \mathrm{MPa} ;$ y $\varepsilon_{\mathrm{R}}=22$, 20 y $19 \%$ respectivamente. En general, puede considerarse que la UTS no varía apenas al ensayar en la dirección longitudinal o en la transversal, pero la ductilidad sí cambia notablemente.

En la aleación Al-4Cr-1Fe (70) se pone de manifiesto que las propiedades mecánicas varían con la temperatura de extrusión, de tal modo que al aumentar la $T_{\text {ext }}$, la dureza y la UTS disminuyen, mientras que la ductilidad y la tenacidad aumentan. Para una aleación Al-4Cr-1Fe extruída a la $T_{\text {ext }}=$ $450{ }^{\circ} \mathrm{C}$, los valores obtenidos a $T_{\mathrm{A}}$ son los siguientes: $Y S=260 \mathrm{MPa} ; U T S=310 \mathrm{MPa} ;$ y $\varepsilon_{\mathrm{R}}=20 \%$. Marshall et al. (72) en la aleación Al-5Cr-2Zr producida, tanto a partir de polvos obtenidos por atomización como de cintas obtenidas por colada de flujo laminar y consolidada por extrusión directa o indirecta, no encuentran grandes diferencias según la procedencia del material, pero sí según el método de extrusión. Así, para una $T_{\text {ext }}=450{ }^{\circ} \mathrm{C}$, se tienen los siguientes valores, respectivamente, según se trate de una extrusión directa o indirecta: para el material procedente de atomización, $Y S=390$ y 345 $\mathrm{MPa} ; U T S=400$ y $350 \mathrm{MPa} ;$ y $\varepsilon_{\mathrm{R}}=17$ y $18 \%$; y para el procedente de CFL, $Y S=400$ y $350 \mathrm{MPa}$;
$U T S=415$ y $387 \mathrm{MPa} ;$ y $\varepsilon_{\mathrm{R}}=19$ y $20 \%$. En ensayos a temperaturas elevadas se produce una pérdida de propiedades, de modo que los valores del $Y S$ y de la UTS bajan hasta 121 y $131 \mathrm{MPa}$ para el material procedente de atomización y 117 y $127 \mathrm{MPa}$ para el procedente de CFL. La tenacidad toma valores entre 11 y $18 \mathrm{MPa} \mathrm{m}^{1 / 2}$.

Por otro lado, Silva et al. (65) han estudiado las propiedades de la aleación Al-5Cr-3Zr(-1Mn) obtenida, tanto por atomización como por fragmentación de cintas procedentes de CFL. La consolidación se realizó mediante desgasificación, compactación en caliente y laminación en caliente, en distintas condiciones. Para los materiales procedentes de polvos atomizados se obtiene, a $T_{\mathrm{A}}$, un valor de $U T S=510$ MPa con un $\varepsilon_{R}=14 \%$, mientras que para los procedentes de partículas de cintas se llega a $540 \mathrm{MPa}$ y 9 $\%$. Los mismos materiales ensayados a $316{ }^{\circ} \mathrm{C}$ dan para los polvos $275 \mathrm{MPa}$ y $6 \%$, y para las cintas pulverizadas $310 \mathrm{MPa}$ y $11 \%$.

Lieblich et al. (73) ponen de manifiesto la influencia del tamaño de partícula, $D$, y de las condiciones de consolidación de la aleación SR Al$5 \mathrm{Cr}-2 \mathrm{Zr}$ sobre la dureza y las propiedades de tracción a $T_{\mathrm{A}}$. Al pasar de un $D=100-200 \mu \mathrm{m}$ a $D<25$ $\mu \mathrm{m}$ se consigue un aumento de la dureza, de la UTS y del $Y S$ de un $24 \%$, lográndose valores de hasta $U T S=391 \mathrm{MPa}, Y S=383 \mathrm{MPa}$, con $\varepsilon_{\mathrm{R}}=12 \%$. Con un tratamiento térmico adecuado, estos valores pueden pasar a $409 \mathrm{MPa}, 394 \mathrm{MPa}$ y $11 \%$, respectivamente. Tanto la UTS como el $Y S$ y la dureza muestran una relación de tipo Hall-Petch respecto del $D$. Las probetas procedentes de $D<25 \mu \mathrm{m}$ presentan una gran respuesta por envejecimiento a 375 ó $400{ }^{\circ} \mathrm{C}$, y la dureza puede pasar de $120 \mathrm{HV}$ hasta casi $160 \mathrm{HV}$.

\subsubsection{Fluencia de las aleaciones $\mathrm{Al}-\mathrm{Cr}-\mathrm{Zr}$}

El comportamiento en fluencia de estos materiales es poco conocido. Mishra et al. (78) han estudiado la fluencia de las aleaciones $\mathrm{Al}-\mathrm{Cr}-\mathrm{Zr}$ en el rango de temperaturas de 315 a $467{ }^{\circ} \mathrm{C}$ utilizando tensiones sumamente bajas. Comparando sus resultados con los de Legzdina et al. en la aleación Al$8,8 \mathrm{Fe}-3,7 \mathrm{Ce}(91)$, encuentran que su resistencia a la fluencia es al menos tan buena como la de esta última aleación. Puesto que la velocidad de fluencia del $\mathrm{Al}-\mathrm{Cr}-\mathrm{Zr}$ a bajas tensiones es varios órdenes de magnitud inferior a la predicción teórica no queda claro cual es el mecanismo de fluencia que da lugar a estos resultados, si bien la energía de activación de la fluencia es del mismo orden que la energía de autodifusión en fronteras de grano, así como el valor del exponente de la tensión se hace igual a la unidad. Además, los valores de la tensión umbral, 
$\sigma_{0}$ no exhiben ninguna dependencia sistemática con la temperatura.

El comportamiento en fluencia de estas aleaciones a cargas mayores, que son las importantes desde un punto de vista tecnológico, ha sido investigado por Brahmi et al. (79). En un estudio de la aleación Al-5Cr-2Zr, procesada con $D<25 \mu \mathrm{m}$ y $T_{\text {ext }}$ $=450{ }^{\circ} \mathrm{C}$, realizado mediante ensayos de tracción con cambios en la velocidad de deformación entre

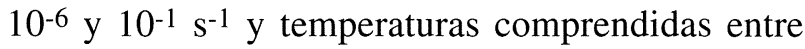
$T_{\mathrm{A}}$ y $550{ }^{\circ} \mathrm{C}\left(0,32-0,88 T_{\mathrm{f}}\right)$ (Fig. 4), encuentran que el comportamiento de la aleación puede describirse mediante la ley potencial de la fluencia. Se distinguen tres intervalos de temperatura bien diferenciados: uno de baja temperatura, desde $T_{\mathrm{A}}$ hasta 250 ${ }^{\circ} \mathrm{C}$, caracterizado por altos valores del exponente de la tensión, $n>20$, y con una energía de activación $Q_{\mathrm{c}}=77 \mathrm{~kJ} \mathrm{~mol}^{-1}$, muy próxima a la de difusión del aluminio a lo largo de las dislocaciones (92). Sin embargo, el alto valor de $n$ no puede asociarse con ningún mecanismo de fluencia controlado por difusión. Los altos valores de $n$ parecen corresponder a los de aleaciones endurecidas por dispersión (93 y 94). Este problema se soluciona introduciendo una tensión umbral $\sigma_{0}(95)$, puesto que la subestructura de la aleación era invariante, tal y como revelaron medidas de dureza (73 y 75). Por tanto, en este intervalo de temperaturas, el comportamiento de la aleación se racionaliza usando el modelo de subestructura constante (96) con un $n=10$ y una tensión umbral, $\sigma_{0}$. El segundo intervalo corresponde a temperaturas intermedias, desde 300 a $400{ }^{\circ} \mathrm{C}$, y está caracterizado por valores de $n$ próximos a 8 y $Q_{\mathrm{c}}=167 \mathrm{~kJ} \mathrm{~mol}^{-1}$, valor muy próximo al de la energía de activación de la autodifusión del aluminio a través de la red (93).

De acuerdo con la literatura (91, 94 y 97-100), la fluencia de un gran número de aleaciones de alumi-

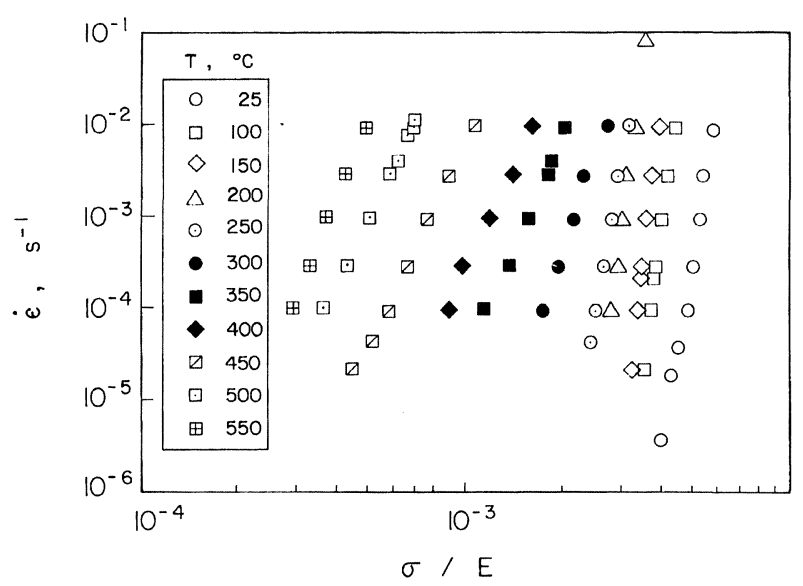

FIG. 4. - Velocidad de deformación frente a la tensión compensada por el módulo de Young.

FIG. 4.- Strain rate vs Young's modulus compensated flow stress. nio endurecidas por dispersión en este mismo intervalo de temperaturas está controlada por la autodifusión del aluminio a través de la red, siendo los valores de $n$ y $Q_{\mathrm{c}}$ concordantes con el modelo de fluencia de subestructura constante (96). El tercer intervalo es el de alta temperatura, desde 450 a 550 ${ }^{\circ} \mathrm{C}$, con $n$ próximo a 8 y $Q_{\mathrm{c}}=273 \mathrm{~kJ} \mathrm{~mol}^{-1}$. El valor de $n$ sugiere que la fluencia podría también ser explicada mediante el modelo de subestructura constante, pero la energía de activación es mucho mayor que la correspondiente a la autodifusión del aluminio, lo que se atribuye a una degradación del material debida a un crecimiento de los precipitados (95). También estudiaron los efectos de las variables de procesado $(D)$ y de los parámetros de extrusión $\left(T_{\text {ext }}\right.$ y $\left.R E\right)$ sobre la microestructura, propiedades mecánicas y comportamiento en fluencia a temperaturas comprendidas entre 100 y $500{ }^{\circ} \mathrm{C}$ (80). Se encontró que el $Y S$ y la UTS dependen fuertemente de la temperatura hasta $400{ }^{\circ} \mathrm{C}$. Cuanto mayores son el $D$ y la $T_{\text {ext }}$, menores son los valores del YS y de la UTS. La tensión de fluencia más alta sé obtiene con muestras procesadas con el menor $D$ a la $T_{\text {ext }}$ más baja. Al aumentar la temperatura de ensayo el efecto benéfico del menor $D$ prevalece sobre el de una baja $T_{\text {ext }}$.

El reforzamiento de la aleación SR Al-5Cr-2Zr está asociado principalmente con el tamaño de grano fino a través de un mecanismo de tipo HallPetch, puesto que éste, a $100{ }^{\circ} \mathrm{C}$, es responsable del $90 \%$ del valor del $Y S$ en todas las extrusiones a $350{ }^{\circ} \mathrm{C}$, con $\sigma_{\mathrm{H}-\mathrm{P}}=k d^{-1 / 2}=(0,90 \pm 0,03) Y S(80)$. La débil contribución de Orowan, a pesar de la gran fracción de volumen de precipitados, se considera razonable, debido a que la mayor parte de los precipitados de $\mathrm{Al}_{13} \mathrm{Cr}_{2}$ están localizados en las fronteras de grano (78), por lo que no se espera de ellos que den ningún refuerzo importante por dispersión. Además, los precipitados $\mathrm{Al}_{3} \mathrm{Zr}$ localizados en el interior de los granos son muy pequeños y cizallables, por lo que su contribución al refuerzo mediante Orowan puede despreciarse (101). Al aumentar la temperatura de ensayo, aunque tiene lugar una degradación considerable en el material, algo de refuerzo persiste incluso a $500{ }^{\circ} \mathrm{C}$. Los resultados obtenidos están de acuerdo con los de Frazier y Koczak (102) y Premkumar et al. (103), según los cuales un mecanismo de tipo Hall-Petch es el refuerzo principal en aleaciones atomizadas de AlTi y Al-Fe-Ni.

En el intervalo de bajas temperaturas existe una $\sigma_{0}$ que disminuye al aumentar la temperatura de ensayo. Esta $\sigma_{0}$ suele relacionarse con el mecanismo de tipo Orowan (104), pero en ocasiones está asociada con el mecanismo de interacción dislocación-partícula (105) o con el trepado local (106). Todos estos mecanismos, junto con el propuesto por Mishra et al. (107) sólo predicen una débil 
dependencia de $\sigma_{0}$ con la temperatura y, por lo tanto, no pueden explicar su comportamiento en el $\mathrm{Al}-5 \mathrm{Cr}-2 \mathrm{Zr}$. En un trabajo posterior (18) se pone de manifiesto que la magnitud de $\sigma_{0}$ en la aleación Al$5 \mathrm{Cr}-2 \mathrm{Zr}$ depende fuertemente del $D$, que está directamente asociado con el tamaño de grano: cuanto mayor es $D$ menor es $\sigma_{0}$. El tamaño de grano afecta a la $\sigma_{0}$ del mismo modo que afecta al $Y S$, lo que sugiere que la $\sigma_{0}$ está asociada con un mecanismo de tipo Hall-Petch. Un resultado parecido se deduce del trabajo de Ehrström y Pineau (101), para una aleación SR Al-Fe.

\section{CONCLUSIONES}

Basándose en el estudio bibliográfico realizado pueden destacarse las siguientes conclusiones:

- Las aleaciones $\mathrm{Al}-\mathrm{Cr}-\mathrm{Zr}$ obtenidas por métodos de solidificación rápida presentan las propiedades mecánicas y la estabilidad térmica necesaria para su utilización en aplicaciones industriales a temperaturas entre 100 y $300{ }^{\circ} \mathrm{C}$, lo que las convierte en buenas candidatas para sustituir en este rango de temperaturas a aleaciones de titanio en un futuro próximo.

- Por razones económicas parece que el método más adecuado para su obtención es la atomización por gas inerte y la posterior extrusión. La técnica del temple desde la fase vapor, aunque permitiría alcanzar mejores propiedades, no parece muy práctica para la producción industrial.

- Antes de que se pueda pasar a la producción industrial de este tipo de aleaciones y a su aplicación en la industria aeronáutica es necesario estudiar otras propiedades importantes, tales como fluencia, ductilidad, tenacidad, fatiga, velocidad de propagación de grietas, resistencia a la corrosión, etc., después de tratar el material a temperaturas y durante tiempos equivalentes a los que se supone han de soportar en servicio.

\section{Agradecimientos}

Los autores desean manifestar su agradecimiento a la Comisión Interministerial de Ciencia y Tecnología (MAT 94-0779) por la financiación otorgada. Asimismo, A. Brahmi agradece a la Comisión de la Unión Europea el disfrute de una beca postdoctoral en el CENIM dentro del programa Human Capital and Mobility (Contact No. ERBCHBGCT 930303).

\section{REFERENCIAS}

(1) CAHN, R.W. Fronteras Cienc. Tecnol., 2, 1993: 12-17.

(2) Millan, P.P. J. Met., 1983: 76.

(3) Palmer, I.G., Thomas, M.P. y Marshall, G.J. Symposium on Dispersion Strengthened Aluminium Alloys. AIME. Phoenix, Arizona (EE.UU.), 1988: 2.001.

(4) Palmer, I.G. y Thomas, M.P. Metallwissenschaft Technik., 41 (6), 1987: 600-605.

(5) Froes, F.H. y Pickens, J.R. J. Met., 1984: 14-28.

(6) Barbaux, Y. y Pons, G. New Rapidly Solidified Aluminium Alloys for Elevated Temperature Applications on Aerospace Structures. EUROMAT 93. París, 1993.

(7) BRITE-EURAM BREU-0356-C "New High Temperature Aluminum Alloys Development".

(8) Rapidly Solidified (RS) Aluminium Alloys. Status y Prospects-National Materials Advisory BoardPublication NMAB. National Academic Press. Washington, 1981.

(9) Aluminium and Aluminium Alloys. ASM Specialty Handbook. $2^{\mathrm{a}}$ ed. ASM International. Materials Park, OH (EE.UU.), 1994: 5.

(10) Tietz, T.E. y Palmer, I.G. ASM Materials Science Seminar. Louisville, K (EE.UU.), Oct. 1981: 189-224.

(11) Adam, C.M. Rapidly Solidified Amorphous and Crystalline Alloys. Materials Research Society, 8, 1982: 411-422.

(12) Jones, H. Rapid Solidifications Processing Principles and Technologies II. Boston, VA. (EE.UU.), Mar. 1980: 306-316.

(13) JoNes, H. Aluminium, 54, 1978: 274.

(14) Gilman, P.S. y Benjamin, J.S. Ann. Rev. Mat. Sci., 13, 1983: 279-300.

(15) Polkin, I.S., Borzov, A.B., Froes, F.H. y Suryanarayana, C. Proc. 2nd Int. Conf. on Mechanical Alloying for Structural Applications. Ed. Barbadillo, J.J., Froes, F.H. y Schwarz, R. ASM International. Vancouver (Canadá), Sep. 1993: 157-164.

(16) Sater, J.M., JHa, S.C. y Sanders Jr., T.H. Aluminum Alloys Contemporary Research and Applications. Ed Vasudevan, A.K. y Doherty, R.D. Academic Press, Inc., 31, 1989: 409-444.

(17) Michot, G. y Champier, G. Rev. Métall. Paris, Jul.Ago., 1991: 426-439.

(18) Brahmi, A., Gerique, T., Torralba, M. y Lieblich, M. Mater. Sci. Eng. A 246, 1998: 55-60.

(19) Ando, T., LaVernia, E.J. y Grant, N. J. Matter Lett., 5 (12), 1986: 17-28.

(20) Zhou, J., Duszczyk, J. y Korevaar, B.M. J. Mat. Sci., 26, 1991: 3.292-3.304.

(21) Bickerdike, R.L., Clark, D., Eastabrook, J.N., Hughes, G., Mair, W.N., Partridge, P.G. y Ranson, H.C. Int. J. Rapid Solidification, 2, 1986: 1-19.

(22) Bickerdike, R.L., Clark, D., Hughes, G., McConnell, M.C., Mair, W.N., Partridge, P.G. y Viney, B.W. Proc. Conf. on Rapidly Solidified Materials. ASM. San Diego, CA (EE.UU.), Sep. 1986: 145-151.

(23) IOANNIDIS, E.K., MARShALl, G.J. y ShePPARD, T. Mater Sci. Technol., 5, 1989: 56-64.

(24) Griffith, W.M., Sanders, R.E. y Hildeman, G.J. Proc. Conf. on High Strength Powder Aluminium Alloys. Dallas, TX (EE.UU.), Feb. 1982: 209-224. 
(25) Palmer, I.G. y Miller, W.S. High Temperature Aluminium Alloys. ASM-Westec, 1986.

(26) Hughes, I.R., Marshall, G.J. y Miller, W.S. Fifth Int. Conf. on Rapidly Quenched Metals. Würzburg (R.F.A.), Sep. 1987: 1.743-1.746.

(27) Midson, S.P., Buckley, R.A. y Jones, H. Rapidly Quenched Metals. Ed. Steeb, S. y Warlimont, H. Elsevier. Nueva York (EE.UU.), 1985: 923-926.

(28) Torralba, M., Sanchez, M.B., Cristina, M.C., Caruana, G. y Garcia-Escorial, A. Proc. 2nd European Conference on Avanced Materials and Processes. Vol. 1. Cambridge. (R.U.), Jul. 1991: 57-63.

(29) Pickens, J.R. Metal Handbook. 10 ed. 2, ASM International. Metal Park, OH (EE.UU.), 1990: 200-215

(30) Lavernia, E.J., Ayers, J.D. y Srivatsan, T.S. Int. Mater. Rev., 37, 1992: 1-44.

(31) Dobatkin, V.I. y Elagin, V.I. Sov. Non-Ferreous Met., 7 (8), 1966: 89-93.

(32) LUBANSKA, H. J. Met., 22 (2), 1970: 45-49.

(33) Imanow, C., Gorczyca, S. y Przybylowicz, K. Arch. Hunt., 14 (4), 1972: 315-320.

(34) Elagin, V.I. y Federov, V. Russ. Metall., 5, 1977: 193197.

(35) Dahl, W., Gruhl, W., Burchard, W.G., Ibe, G. y DuMitrescu, C. Z. Metallkd., 68, 1977: 121-127.

(36) Dahl, W., Gruhl, W., Burchard, W.G., Ibe, G. y DuMitRESCU, C. Z. Metallkd., 68, 1977: 188-194.

(37) SAhin, E. y Jones, H. Proc. 3rd Int. Cong. on Rapidly Quenched Metals. Ed. Cantor, B. The Metals Society. Londres (R.U.), 1, 1978: 138-146.

(38) PARK, W.W. y KIM, T.H. J. Korean Inst. Met., 22, 1984: 721-728.

(39) KIM, P.K. y Kiм, T.H. J. Korean Inst. Met., 22, 1984: 521-528.

(40) HaN, S.H. y KIM, T.H. J. Korean Inst. Met., 23, 1985: 611-618.

(41) PArK, W.W. y Kim, T.H. Proc. Conf. Korean-Japan Proc. on Rapid Solidification Technology. ASM International. Metals Park, OH (EE.UU.), 1986: 28-32.

(42) KIM, J.R. y KIM, T.H. J. Korean Inst. Met., 1987, 25, 1988: 506-513.

(43) Hughes, I.R., Marshall, G.J. y Miller, W.S. Rapidly Quenched Metals. Ed. Steeb, S. y Warlimont, H. Elsevier. Nueva York (EE.UU.), 1985: 1.743-1.746.

(44) Marshall, G.J., Hughes, I.R. y Miller, W.S. Mater. Sci. Technol., 2 (4), 1986: 394-399.

(45) Miller, W.S., Hughes, I.R., Palmer, I.G., Thomas, M.P., Saini, T.H. y White, J. High Strength Powder Metallurgy Aluminium Alloys II. Ed. Hildeman, G.J. y Koczak, M.J. TMS. Warrendale, PA (EE.UU.), 1986: 311-331.

(46) Saunders, N. y Rivlin, V.G. Mater. Sci. Technol., 2 (6), 1986: 521-527.

(47) Bendersky, L., Schaefer, R.J., Biancaniello, F.S. y SheChtMan, D. J. Mater. Sci., 21, 1986: 1.889-1.896.

(48) McConnell, M.C. y PArtridge, P.G. Acta Metall., 35 (8), 1987: 1.973-1.980.

(49) Partridge, P.G. y McConnell, M.C. Acta Metall., 35 (8), 1987: 1.981-1.993

(50) Thomas, M.P., Palmer, I.G. y Baker, C. Processing of Structural Metals by Rapid Solidification. Ed. Froes, F.H. y Savage, S.J. ASM International. Metal Park, OH (EE.UU.), 1987: 337-345.
(51) Miller, W.S. y Palmer, I.G. Met. Powder Rep., 41, 1986: 761-767.

(52) Palmer, I.G., Thomas, M.P. y Marshall, G.J. Dispersion Strengthened Aluminium Alloys. Ed. Kim., Y.-W. y Griffth, W.J. TMS. Warrendale, PA (EE.UU.), 1988: 217-241.

(53) ParK, W.W. y Kim, T.H. Scripta Metall., 22 (11), 1988: 1.709-1.714.

(54) Tsakiropoulos, P., Pratt, R., Jones, H., Restall, J.E. y Gardiner, R.W. Mater. Sci. Eng., A98, 1988: 143147.

(55) Adkins, N.J.E., Saunders, N. y Tsakiropoulos, P. Mater. Sci. Eng., A98, 1988: 217-219.

(56) Saunders, N. y Tsakiropoulos, P. Mater. Sci. Technol., 4 (2), 1988: 157-160.

(57) Pan, L.-M., Saunders, N. y Tsakiropoulos, P. Mater. Sci. Technol., 5 (6), 1989: 609-612.

(58) Adkins, N.J.E., Saunders, N. y Tsakiropoulos, P. Mater. Sci. Eng., A133, 1991: 767-770.

(59) Adkins, N.J.E. y Tsakiropoulos, P. Mater. Sci. Eng., A134, 1991: 1.158-1.161.

(60) Adkins, N.J.E. y Tsakiropoulos, P. Mater. Sci. Tech., 7 (4), 1991: 334-340.

(61) Adkins, N.J.E. y Tsakiropoulos, P. Mater. Sci. Technol., 7 (5), 1991: 419-426.

(62) Tezcan, R. y Jones, H. Mater. Lett., 10 (6), 1990: 231234.

(63) Silva, M.P., Jones, H. y Sellars, M.C. Proc. Conf. PM 90. The Institute of Metals. Londres (R.U.), 2, 1990: 311-314.

(64) Silva, M.P., Whiteman, J.A., Jones, H. y Sellars, M.C. Proc. Conf. PM 90. The Institute of Metals. Londres (R.U.), 2, 1990: 315-319.

(65) Silva, M.P., Jones, H. y Sellars, M.C. Mater. Sci. Eng., A134, 1991: 1.107-1.110.

(66) Chung, M.S., Tu, G.C. y Chen, I.T. J. Mater. Sci., 23, 1991: 268-277.

(67) Tu, G.C., Chen, I.T. y Chung, M.S. J. Mater. Sci. Lett., 10, 1991: 1.125-1.128.

(68) IOANnidis, E.K. y ShePPard, T. Mater. Sci. Technol., 6 (6), 1990: 528-434.

(69) IoAnNidis, E.K. y ShePpard, T. Mater. Sci. Technol., 6 (9), 1990: 749-754.

(70) IOANNIDIS, E.K. y SHEPPARD, T. J. Mater. Sci., 25, 1990: 3.965-3.975

(71) IOANNIDIS, E.K. y SHEPPARD, T. J. Mater. Sci., 26, 1991: 4.795-4.802.

(72) Marshall, G.J. y Ioannidis, E.K. J. Mater. Sci., 27, 1992: 3.552-3.564.

(73) Lieblich, M., Caruana, G., Torralba, M. y Jones, H. Int. J. Rapid Solidification, 9 (1), 1995: 45-61.

(74) Lieblich, M., Caruana, G., Torralba, M. y Jones, H. Mater. Sci. Technol., 12 (1), 1996: 25-33.

(75) Caruana, G., Lieblich, M., Torralba, M. y Jones, H. J. Mater. Sci. Letters., 15, 1996: 568-570.

(76) Lieblich, M., Caruana, G., Torralba, M. y Jones, H. Powder Metall., 39 (2), 1996: 143-149

(77) Lieblich, M., Caruana, G., Torralba, M. y Jones, H. European Conference on Advanced Powder Metallurgy Materials (EURO PM95). Birmingham (R.U.), Oct. 1995: 240-247.

(78) Mishra, R.S., Jones, H. y Greenwood, G.W. Int. J. Rapid. Solidification, 5, 1990: 149-162. 
(79) Brahmi, A., Gerique, T., Torralba, M. y Lieblich, M. Scr. Materialia, 35 (12), 1996: 1.449-1.454.

(80) Brahmi, A., Gerique, T., Torralba, M. y Lieblich, M. Scr. Materialia, 37 (11), 1997: 1.623-1.629.

(81) Boettinger, W.J., Bendersky, L. y Early, J.G. Metal. Trans., 17A, 1986: 781-790.

(82) Kamio, A., Tezuka, H., Sato, T., Long, T.T. y TAKAHASHI, T.J. Jpn. Inst. Light Met., 36, 1986: 72-80.

(83) Kim, K.B., Cha, J.M., Cho, S.H., WeE, D.M., NAm, T.Y. y Yood, E.P. K. Korean Inst. Met., 23, 1985: 697705.

(84) Hansen, M. Constitution of Binary Alloys. 2th ed. Ed. McGraw-Hill Book Company, Inc. Nueva York (EE.UU.), 1958: 81-84 y 152-154.

(85) Adkins, N.J.E. y Tsakiropoulos, P. Int. J. Rapid Solidification, 6, 1991: 101-114.

(86) Shao, G. y Tsakiropoulos, P. Acta Metall. Mater., 42 (9), 1994: 2.937-2.942.

(87) Marshall, G.J. J. Mater. Sci., 22, 1987: 3.581-3.588.

(88) Adkins, N.J.E., y Tsakiropoulos, P. Int. J. Rapid Solidification, 6, 1991: 87-100.

(89) Sanchez, B., Garcia-Escorial, A., Cristina, M.C., Caruana, G. y Torralba, M. Mater. Sci. Technol., 12 (10), 1996: 794-801.

(90) Jones, H. J. Mater. Sci., 19, 1984: 1.043-1.076.

(91) Legzdna, D. y Parthasarathy, T.A. Metall. Trans., 18A, 1987: 1.713-1.719.

(92) Frost, H.J. y Ashby M.F. Deformation Mechanism Maps. Ed. Pergamon Press. Londres (R.U.), 1982: 21.
(93) Lin, J. y SHERBy, O.D. Res. Mechanica, 2, 1981: 251-293.

(94) Carreño, F., Gonzalez-Doncel, G. y Ruano, O.A. Mater. Sci. Eng., A164, 1993: 216-219.

(95) Luthy, H., Miller, A.K. y Sherby, O.D. Acta Metall., 28, 1980: 169-178.

(96) Sherby, O.D., Klundt, R.H. y Miller, A.K. Metall. Trans., 8A, 1977: 843-850.

(97) Mishra, R.S., ParadKar, A.J. y RaO, K.N. Acta Metall. Mater., 41, 1993: 2.243-2.251.

(98) Legzdina, D. y Parthasarathy, T.A. Metall. Trans., 21A, 1990: 2.155-2.158.

(99) Wolfenstine, J., Gonzalez-Doncel, G. y Sherby, O.D. J. Mater. Sci. Letters, 9, 1990: 410-413.

(100) Yeh, Y.H., NaKashima, H., KuRishita, H., Goto, G. y YoshinAGA, H. Mater. Sci. JIM, 31, 1990: 284-292.

(101) Ehrström, J.C. y Pineau, A. Mater. Sci. Eng., A186, 1994: 55-64.

(102) Frazier, W.E. y KocZaK, M.J. Dispersion Strengthened Aluminum Alloys. Ed. Kim, Y.W. y Griffith, W.M. TMS. Warrendale, PA (EE.UU.), 1988: 573-602.

(103) Premkumar, M.K., Lawley, A. y KoczaK, M.J. Mater. Sci. Eng., A174, 1994: 127-139.

(104) Lund, R.W. y Nix, W.D. Acta Metall., 24, 1976: 469481.

(105) Arzt, E. y Grahle, P: Z. Metallkd., 87, 1996: 874-884.

(106) Shewfelt, R.S.W. y Brown, L.M. Phil. Mag., 35, 1977: 945-962.

(107) Mishra, R.S., Nandy, T.K. y Greenwood, G.W. Phil. Mag., 69A, 1994: 1.097-1.109. 